\title{
Transient Pressure Analysis for Multi-Fractured Horizontal Well with the Use of Multi-Linear Flow Model in Stress Sensitive Shale Gas Reservoir
}

\author{
Guangdong Wang a ${ }^{a}$ Ailin Jia $^{a}$, Yunsheng Wei ${ }^{a}$, Cong Xiao ${ }^{b}$ \\ a Research Institute of Petroleum Exploration and Development, CNPC, 100083, China \\ belft Institute of Applied Mathematics, Delft University of Technology, Mekelweg 4, 2628 CD Delft, \\ the Netherlands
}

\begin{abstract}
Shale gas reservoirs (SGR) are important replacements for conventional energy resources and have been widely exploited by hydraulic fracturing technologies. On the one hand, due to the inherent ultra-low permeability and porosity, there is stress sensitivity in the reservoirs generally. On the other hand, hydraulic fractures and the stimulated reservoir volume (SRV) generated by the massive hydraulic fracturing operation have contrast properties with the original reservoirs. These two phenomena bring huge challenges in SGR transient pressure analysis.

Although some works in the literatures have been done on the transient pressure analysis of multi-fractured horizontal wells in SGR, unfortunately, none of them has taken the stress sensitivity and spatially varying permeability of SRV zone into consideration simultaneously. To fill this gap, this paper first idealizes the SGR to be four linear composite regions. What's more, SRV zone is further divided into sub-sections on the basis of non-uniform distribution of proppant within SRV zone which easily yields spatially varying permeability away from the main hydraulic fracture. The stress sensitivity is characterized by the varying permeability depended on the pore pressure. By means of perturbation transformation and Laplace transformation, an analytical multi-linear flow model (MLFM) is obtained and validated by the comparison with the previous model. On the basis of our model, the flow regimes are identified and the sensitivity analysis of critical parameters are conducted to further understand the transient pressure behaviors. The research results provided by this work are of significance for well test interpretation and production performance analysis of SGR.
\end{abstract}

Key words: shale gas reservoirs; stress sensitivity; multi-fractured horizontal well; spatially varying permeability; pressure transient analysis

\section{Abbreviations:}

SGR, shale gas reservoir; SRV, stimulated reservoir volume; MLFM, multi-linear flow model.

\section{Introduction}

Due to extremely low permeability and porosity of shale gas reservoir (SGR), multistage hydraulic fracturing has become an integral tool to improve the gas recovery. The economic feasibility of shale gas reservoirs has a strong relationship with the fracture system permeability near the wellbore. Considered to be the most effective way to produce gas resources, multistage fractured horizontal well can create several high-conductivity hydraulic fractures as flow paths, at the same time, activate and connect existing natural fractures so as to develop large fracture network system (Clarkson, 2013). The zone containing the main high-conductivity hydraulic fractures and large spatial network system which can effectively 
improve well performance is defined as SRV (stimulated reservoir volume), and the remaining zone which hardly influenced by the treatment of hydraulic fracturing is similarly defined as USRV (un-stimulated reservoir volume) (Ozkan et al.,2011; Mayerhofer et al., 2006).

The presence of a complex fracture network in the SRV has a significant impact on the pressure transient analysis of unconventional reservoirs. Analytical and semi-analytical approaches have been used to model the transient flow behavior in such systems. These models can be divided into two categories according to the conductivity of hydraulic fractures: (1) Radial flow model. These models assume that there is no pressure drop along the hydraulic fractures and the pressure is equal to bottom-hole pressure. In another words, the hydraulic fractures can be regarded as the parts of the wellbore. Zhao et al $(2014,2016)$ and Wang (2013) have established semi-analytical solutions with the use of Laplace transformation. The point source function or line source function, coupled with superposition principle,was utilized to mathematically incorporate the interference among hydraulic fractures. (2) Multi-Linear flow model. Similarly, these models assumed that there is continuous pressure drop along the hydraulic fractures from the tip of hydraulic fractures to the wellbore. El-Banbi (1998), Hasan and Al-Ahmadi (2011), Xu et al (2012), Ekaterina Stalgorova (2012) simplified the shale gas reservoirs as linear composition reservoirs and the governing equations of each zone can be derived, however, the interference among hydraulic fractures is ignored. Besides, some experimental techniques and theoretical methods on the stress sensitive media have been presented, given out important parameters and models to describe stress-sensitive effect. Fatt and Davis (1952); Fatt,0 and Gray (1953) studied the effect of stress sensitivity on permeability and porosity via experimental study for typical rocks under tri-axial stress state and found that porosity is less sensitive to pressure drop in the formation compared with permeability. Vairogs et al. (1973) performed more detailed stress-sensitive experiments on rock samples with different initial permeability and concluded that there is greater degree of permeability reduction under low permeability conditions. Pedrosa.O.A (1986) introduced the permeability modulus to establish an equation about the relationship between the permeability and the pressure drop. Kilmer et al (1987) and Han et al (2013) carried out researches on stress sensitivity tight reservoirs by using indoor experimental measures and provided the relational expression for the various permeability and the effective pressure. The above fundamental research results provided powerful tools to studied the effect of stress sensitivity on transient pressure response and rate performance of fractured horizontal well in low permeable reservoirs.

To address these weaknesses, many semi-analytical models without such simplifications have been proposed by treating the reservoirs after hydraulic fracturing as radial composite region. Zhao et al. (2016) simplified the SRV into a circular high permeability area and used the radial composite model to study the pressure behaviors of fractured wells in unconventional reservoirs. Many useful point source and linear source functions in radial composite regions with different boundary conditions were first proposed in their work, although the flow forms of the fluids were no longer limited to be linear, only pressure transient behaviors of horizontal well with fractures vertical to the wellbore is discussed. On the basis of source functions, Jiang et al. (2014) conducted rate and pressure transient analysis for multistage fractured horizontal well in tight oil reservoirs considering SRV, but the fractures were still limited to be perpendicular with the wellbore and stress sensitivity was neglected in their work. Ren 0t al. $(2016,2017)$ combined the linear source function method and the Perturbation technique to take stress sensitivity into consideration when they studied the transient pressure behaviors of horizontal wells, but the complexity of the fracture networks was ignored. Jia et al. (2017) presented a comprehensive model combining finitedifference and boundary-element method for the flow behaviors of volume fractured 
horizontal well. Although the irregular geometry of the complex fracture networks, internal and external boundaries were captured in their model, the stress sensitivity was still neglected and the modeling process was complex due to the comprehensive application of various mathematical methods. Considering the stress sensitivity of fractures and the reservoirs, Wang 0t al. (2017) proposed a semi-analytical model for transient flow behaviors of fractured horizontal well with complex fracture networks in homogeneous tight reservoirs, with no thought for the property difference between the inner SRV and the outer un-stimulated region.

Although semi-analytical radial flow model could be employed to characterize hydraulic fractures with complex topology, the multi-linear flow models are easy to be used in the realfield application. By means of perturbation transformation and Laplace transformation, we proposed a new analytical multi-linear flow model to systematically investigate the effects of stress sensitivity in SGR. In addition, the SRV zone is further divided into sub-sections on the basis of non-uniform distribution of proppant within SRV zone which easily yields spatially varying permeability away from the main hydraulic fracture. This paper also discusses the influence of relevant parameters on the of fractured horizontal wells in stress sensitive SGR, including stress sensitivity, mobility ratio of the SRV and the outer region, SRV size, coefficient of permeability variation. Corresponding solutions can be useful for fracturing design and well test interpretation in field practice.

\section{Mathematical model and analytical solution}

\subsection{Model descriptions}

A simplified physical model of multi-fractured horizontal well in SGR is illustrated in Fig.1(a). The entire reservoir after hydraulic fracturing can be conceptually divided into four coupled linear flow zones with different properties, including main hydraulic fracture zone $\mathbf{I}$, SRV zone II, SRV zone III and the other outer zone without stimulation zone IV. The fracture half-length is $x_{\mathrm{f}}$, the width and length of entire zone are $x_{\mathrm{e}}$ and $y_{\mathrm{e}}$, respectively. The length of SRV zone II is $l$. All these variables have been depicted in Fig.1(a). The initial permeability of these four zones are separately denoted as $k_{1}, k_{2}, k_{3}$ and $k_{4}$. 


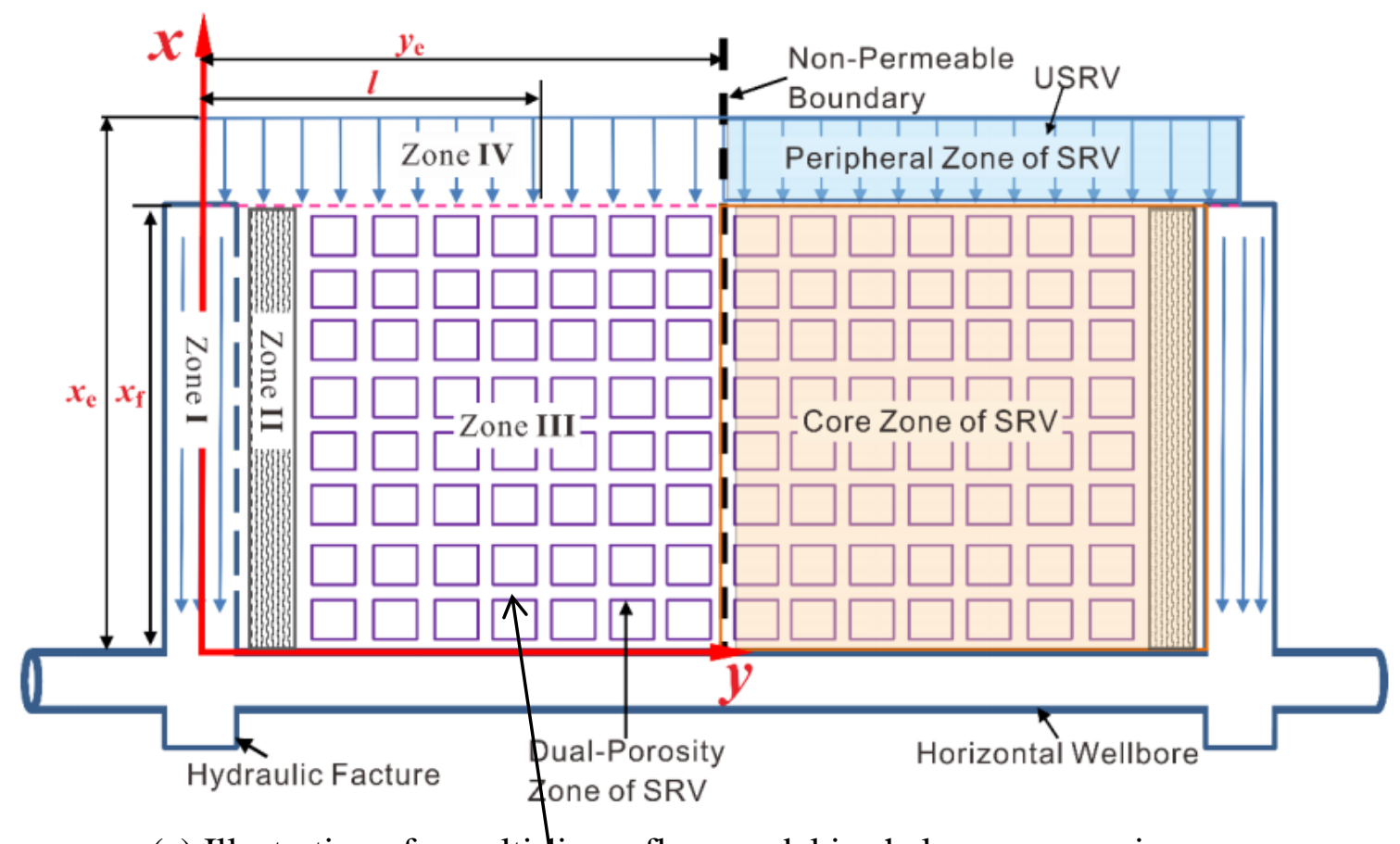

(a) Illustration of a multi-linear flow model in shale gas reservoir

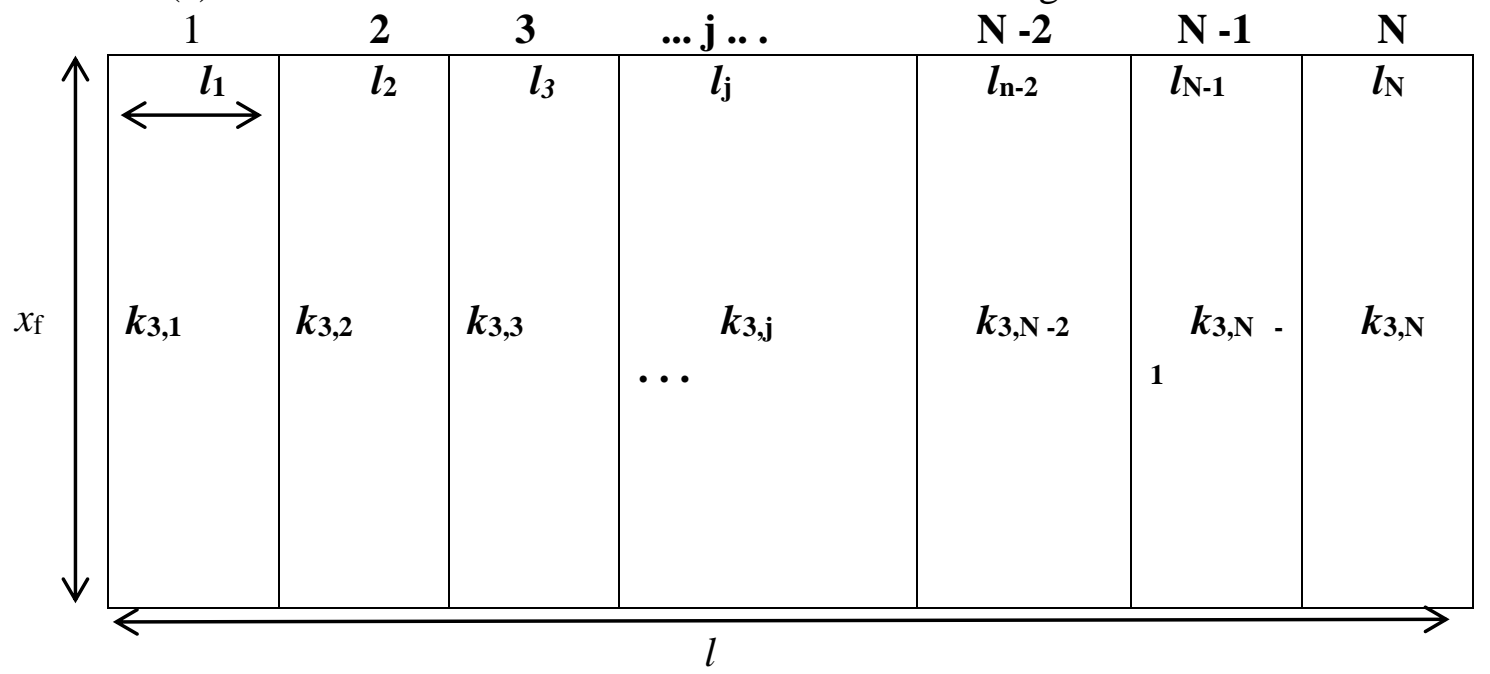

(b) Illustration of a further subdivision of Zone II

Fig.1: Physical model of multi-fractured horizontal well in shale gas reservoir

As far as we known, the non-uniform distribution of proppant within SRV zone easily yields spatially varying permeability away from the main hydraulic fracture. In this paper, we further divide Zone III into $N$ small zones as illustrated in Fig.1(b).

Other basic assumptions are as follows:

(1) All zones are idealized as single porosity media with different permeability.

(2) Only the stress sensitivity of permeability is taken into consideration, while the porosity is assumed to be constant during the production.

(4) The fluid in the whole reservoirs is simplified to be single-phase and microcompressible, and the seepage of the fluid follows the Darcy's Law.

(5) All hydraulic fractures are vertical and fully penetrate the formation, so the flow pattern in this work is simplified into be plane linear flow, ignoring the influence of gravity and capillary force. 
(6) Wellbore have infinite conductivity, the well is produced at a constant rate and the fluids flow towards to the wellbore only via the main hydraulic fractures.

\subsection{Mathematical Formula of Multi-Linear Model}

According to the theory proposed by Pedrosa. O.A. , the pressure-dependent permeability could be expressed as the exponential function of pore pressure, which has:

$$
k_{i}(m)=k_{0} e^{-\gamma_{i}\left(m_{0}-m_{i}\right)}, \quad i=1,2,3,4
$$

Where, the reference permeability $k_{\text {ref. }} k_{0}$ is the initial permeability of fracture system in the reservoir, $\mathrm{m}^{2} ;{ }^{\gamma_{i}}$ is the permeability modulus, which is determined by the property of rock geomechanics and always in the range of $10^{-5} \sim 10^{-4} \mathrm{pa}^{-1}$. We also assume that four zones have different permeability modulus.

The mechanism of adsorption can be classified into instant adsorption and timedependent adsorption, and the former is selected to describe adsorption phenomenon in this paper. At present, Langmuir isotherm equation is to describe the instant adsorption process of shale gas (Chen et al, 2017), and its expression is:

$$
V_{E}=V_{L} \frac{m}{m_{L}+m}
$$

Where, $V_{\mathrm{E}}$ is the adsorption equilibrium concentration, $\mathrm{sm}^{3} / \mathrm{m}^{3} ; V_{\mathrm{L}}$ is the Langmuir adsorption concentration, $\mathrm{sm}^{3} / \mathrm{m}^{3} ; P_{\mathrm{L}}$ is the Langmuir pressure, MPa; $m_{\mathrm{L}}$ is Langmuir pressure, $\mathrm{MPa}^{2} /(\mathrm{mPa} \bullet \mathrm{s})$.

For the convenience and simplicity of formulas deducing, some dimensionless parameters are introduced first.

$$
\begin{gathered}
m_{i}=\frac{k_{r e f} h\left[m_{0}-m_{i}\right]}{1.842 q_{g s c} \mu_{g i} B_{g i}}, i=1,2,3,4 \quad t_{D}=\frac{3.6 \times 10^{-3} k_{r e f} t}{\mu_{g i}\left(\phi C_{g}\right)_{r e f} x_{f}^{2}} \\
x_{D}=\frac{x}{x_{f}}, \quad y_{D}=\frac{y}{x_{f}}, \quad l_{j, D}=\frac{l_{j}}{x_{f}}, \quad w_{f D}=\frac{w_{f}}{x_{f}}, \quad \gamma_{i D}=\frac{1.842 q_{s c} \mu_{g} B_{g}}{k_{r e f} h} \gamma_{i} \\
C_{f D}=\frac{k_{1} w_{f}}{k_{r e f} x_{f}}, \quad k_{i D}=\frac{k_{i}}{k_{r e f}}, \quad \omega_{i D}=\frac{\phi_{i} C_{g i}}{\left(\phi C_{g}\right)_{r e f}} \\
\omega_{3 m D}=\frac{\phi_{3 m} C_{g i}}{\left(\phi C_{g}\right)_{r e f}}, \quad \lambda=\sigma \frac{k_{3 m}}{k_{r e f}} L_{r e f}^{2} \\
m_{D L}=\frac{k_{r e f} h\left[m_{L}-m_{i}\right]}{1.842 q_{g s c} \mu_{g i} B_{g i}}, \quad, \quad V_{D L}=2 \frac{p_{s c} T}{\mu_{i} T_{S C}} \frac{k_{r e f} h\left[m_{L}-m_{i}\right] \phi_{3 m} V_{L}}{1.842 q_{g s c} \mu_{g i} B_{g i}}
\end{gathered}
$$

A multi-linear flow model (MLFM) will be used to derive the mathematical equations. In the following parts, the governing equations are separately established for each zone.

\subsubsection{Zone I}

In this zone, the gas supplies from the adjacent reservoir Zone II to the main hydraulic fractures, and then flow to wellbore with a linear flow pattern. Gas pseudo-pressure is used to consider the effects of gas compressibility. When the stress sensitivity is considered, the governing function can be presented as follows, 


$$
\frac{\partial^{2} m_{1}}{\partial x^{2}}+\gamma_{1}\left(\frac{\partial m_{1}}{\partial x}\right)^{2}+\left.\frac{2}{w_{f}} \frac{k_{2,1}}{k_{1}} \frac{\partial m_{2,1}}{\partial y}\right|_{y=\frac{w_{f}}{2}}=e^{\lambda_{1}\left(m_{0}-m_{1}\right)} \frac{\phi_{1} \mu C_{g}}{k_{1}} \frac{\partial m_{1}}{\partial t}
$$

With the inner and outer boundary conditions:

$$
\left.e^{-\gamma_{1}\left(m_{0}-m_{1}\right)} \frac{\partial m_{1}}{\partial x}\right|_{x=0}=\frac{q_{g s c} \mu_{g i} B_{g i}}{2 w_{f} h k_{1}},\left.\quad \frac{\partial m_{1}}{\partial x}\right|_{x=x_{f}}=0
$$

Substituting predefined dimensionless variables to Eq.(3) and Eq.(4), their dimensionless formula is as follows.

$$
\frac{\partial^{2} m_{1 D}}{\partial x_{D}^{2}}+\gamma_{1 D}\left(\frac{\partial m_{1 D}}{\partial x_{D}}\right)^{2}+\left.\frac{2 k_{2,1 D}}{C_{1 D}} \frac{\partial m_{2,1 D}}{\partial y_{D}}\right|_{y_{D}=\frac{w_{f D}}{2}}=e^{\gamma_{1 D} m_{1 D}} \frac{\omega_{1 D}}{k_{1 D}} \frac{\partial m_{1 D}}{\partial t_{D}}
$$

With $\left.e^{-\gamma_{1 D} m_{1 D}} \frac{\partial m_{1 D}}{\partial x_{D}}\right|_{x_{D}=0}=-\frac{\pi}{C_{1 D}},\left.\quad \frac{\partial m_{1 D}}{\partial x_{D}}\right|_{x_{D}=1}=0$

Eq. (5) is strongly nonlinear partial differential equation, which are not convenient to be solve analytically. A perturbation transformation proposed by Pedrosa O.A. can be used to eliminate the non-linearity. The new dimensionless variables $\eta_{j D}$ related to the dimensionless pressure are introduced as follows:

$$
m_{i D}=-\frac{1}{\gamma_{i D}} \ln \left[1-\gamma_{i D} \eta_{i D}\right], i=1,2,3,4
$$

According to the simplified method proposed by 0 , due to the dimensionless permeability modulus $\gamma_{D}$ is usually a small value, $\eta_{j D}$ can be expanded as a power series in the parameter $\gamma_{D}$.

$$
\begin{aligned}
& \eta_{j D}=\eta_{j D 0}+\gamma_{D} \eta_{j D 1}+\left(\gamma_{D}\right)^{2} \eta_{j D 2}+\left(\gamma_{D}\right)^{3} \eta_{j D 3}+\ldots \\
& \frac{1}{1-\gamma_{D} \eta_{j D}}=1+\gamma_{D} \eta_{j D}+\left(\gamma_{D}\right)^{2}\left(\eta_{j D}\right)^{2}+\left(\gamma_{D}\right)^{3}\left(\eta_{j D}\right)^{3}+\ldots
\end{aligned}
$$

Substituting Eq.(7) and (8) into Eq.(5), we can get a sequence of linear problem that can be solved for $\eta_{j D 0}, \eta_{j D 1}$, and so on. According to Liu et al (2015) the zero-order approximation $\eta_{j D 0}$ was accurate enough for pressure analysis.

$$
\frac{\partial^{2} \eta_{1 D}}{\partial x_{D}^{2}}+\left.\frac{2 k_{2,1 D}}{C_{1 D}} \frac{\partial \eta_{2,1 D}}{\partial y_{D}}\right|_{y_{D}=\frac{w_{f D}}{2}}=\frac{\omega_{1 D}}{k_{1 D}} \frac{\partial \eta_{1 D}}{\partial t_{D}}
$$

With $\left.\frac{\partial \eta_{1 D}}{\partial x_{D}}\right|_{x_{D}=0}=-\frac{\pi}{C_{1 D}},\left.\quad \frac{\partial \eta_{1 D}}{\partial x_{D}}\right|_{x_{D}=1}=0$

Finally, Laplace transformation can be used to transform these equations from time domain to Laplace domain so as to eliminate the effects of time domain.

$$
\frac{d^{2} \overline{\eta_{1 D}}}{d x_{D}^{2}}+\left.\frac{2 k_{2,1 D}}{C_{1 D}} \frac{d \overline{\eta_{2,1 D}}}{d y_{D}}\right|_{y_{D}=\frac{w_{f D}}{2}}=s \frac{\omega_{1 D}}{k_{1 D}} \overline{\eta_{1 D}}
$$

With $\left.\frac{d \overline{\eta_{1 D}}}{d x_{D}}\right|_{x_{D}=0}=-\frac{1}{s} \frac{\pi}{C_{1 D}}$, and $\left.\frac{d \overline{\eta_{1 D}}}{d x_{D}}\right|_{x_{D}=1}=0$ 


\subsubsection{Zone II}

Zone II is a highly permeable SVR zone which is adjacent to the main hydraulic fracture as illustrated in Fig.2(a). This area is significantly stimulated by the massive hydraulic fracturing operation, as a result, it can be assumed to single porous media with high permeability due to the support by the proppant. When the stress sensitivity is considered, the governing function can be presented as follows,

$$
\frac{\partial^{2} m_{2}}{\partial y^{2}}+\gamma_{2}\left(\frac{\partial m_{m_{2}}}{\partial y}\right)^{2}+\left.\frac{k_{4}}{k_{2}} \frac{\partial m_{4}}{\partial y}\right|_{x=\frac{x_{f}}{2}}=e^{\gamma_{2}\left(m_{0}-m_{2}\right)} \frac{\phi_{2} \mu C_{g}}{k_{2}} \frac{\partial m_{2}}{\partial t}, \frac{w_{f}}{2}<x<l_{1}
$$

The left condition is adjacent to Zone I, while the right condition is connected to Zone III. Specifically speaking, these two conditions can be presented.

$$
\left.m_{2}\right|_{y=w_{f} / 2}=\left.m_{1}\right|_{y=w_{f} / 2},\left.\left.\quad m_{2}\right|_{y=l_{1}}\right|_{=}=\left.\left.m_{3}\right|_{y=l_{1}}\right|_{\mid}
$$

Their dimensionless formulas with the consideration of stress sensitivity effects can be as follows

$$
\frac{\partial^{2} \eta_{2 D}}{\partial y_{D}^{2}}+\left.\frac{k_{4 D}}{k_{2 D}} \frac{\partial \eta_{4 D}}{\partial x_{D}}\right|_{x_{D}=1}=\frac{\omega_{2 D}}{k_{2 D}} \frac{\partial \eta_{2 D}}{\partial t_{D}}, w_{f D} / 2<y_{D}<l_{1, D}
$$

With the boundary conditions:

$$
\left.\gamma_{1 D} \eta_{1 D}\right|_{y=w_{f D} / 2}\left|=\gamma_{2 D} \eta_{2}\right|_{y=w_{f D} / 2},\left.\quad \gamma_{2 D} \eta_{2 D}\right|_{y=l_{1, D}}\left|=\gamma_{3,1 D} \eta_{3,1 D}\right|_{y=l_{1, D}}
$$

Similarly, Laplace transformation can be used to transform these equations from time domain to Laplace domain.

$$
\frac{d^{2} \overline{\eta_{2 D}}}{d y_{D}^{2}}+\left.\frac{k_{4 D}}{k_{2 D}} \frac{d \overline{\eta_{4 D}}}{d x_{D}}\right|_{x_{D}=1}=s \frac{\omega_{2 D}}{k_{2 D}} \overline{\eta_{2 D}}, w_{f D} / 2<y_{D}<l_{1, D}
$$

With the boundary conditions:

$$
\left.\gamma_{1 D} \overline{\eta_{1 D}}\right|_{y=w_{f D} / 2}\left|=\gamma_{2 D} \overline{\eta_{2 D}}\right|_{y=w_{f D} / 2},\left.\quad \gamma_{2 D} \overline{\eta_{2 D}}\right|_{y=l_{1, D}}\left|=\gamma_{3,1 D} \overline{\eta_{3,1 D}}\right|_{y=l_{1, D}}
$$

\subsubsection{Zone III}

In this work, Zone III is also assumed to be a stimulated area induced by massive hydraulic fracturing operation. Unlike Zone II, this zone is partially supported by the proppant. In addition, the pre-existing natural fractures are stimulated as well. On condition to these facts, Zone III is idealized as a dual-porosity media where the fracture and matrix are coupled. As we have mentioned above, to systematically investigate the influences of spatially varying permeability away from the main hydraulic fracture, we have divided Zone III into $N$ small zones as illustrated in Fig.2(b). We need to establish flow equations for each small zones and coupled them together on the basis of continuity condition at the interface of neighboring zones. Here, we take the $j$-th small zone as an example to derive a set of generic governing equations. We also should separately derive the governing equations for fracture system and matrix system. The effects of adsorption and pseudo-steady state inter-porosity flow from matrix to micro fracture system are considered simultaneously. When the stress sensitivity is considered, the governing function can be presented as follows

Fracture system: 


$$
\frac{\partial^{2} m_{3, j}}{\partial y^{2}}+\gamma_{3, j}\left(\frac{\partial m_{m_{3, j}}}{\partial y}\right)^{2}+\left.\frac{k_{4}}{k_{3, j}} \frac{\partial m_{4}}{\partial y}\right|_{x=x_{f}}+\sigma \frac{k_{3 m}}{k_{3, j}}\left(m_{3 m}-m_{3, j}\right)=e^{\gamma_{3, j}\left(m_{0}-m_{3, j}\right)} \frac{\phi_{3, j} \mu C_{g}}{k_{3, j}} \frac{\partial m_{3, j}}{\partial t}, l_{j}<x<l_{j+1}
$$

Matrix system:

$$
-\sigma k_{3 m}\left(m_{3 m}-m_{3, j}\right)=\phi_{3 m} \mu C_{g} \frac{\partial m_{3 m}}{\partial t}+\frac{p_{s c} T}{T_{S C}} \frac{\partial V}{\partial t}
$$

The boundary conditions are derived from the interface of neighboring two sections, where the pressure and gas flux should be strictly equal between those two sections.

$$
\left.m_{3, j}\right|_{y=l_{j}}=\left.m_{3, j+1}\right|_{y=l_{j}},\left.\quad k_{3, j} e^{-\gamma_{3, j}\left(m_{0}-m_{3, j}\right)} \frac{\partial m_{3, j}}{\partial y}\right|_{y=l_{j}}=\left.k_{3, j+1} e^{-\gamma_{3, j+1}\left(m_{0}-m_{3, j+1}\right)} \frac{\partial m_{3, j+1}}{\partial y}\right|_{y=l_{j}}, j=2, \ldots, N-1
$$

Especially, the first small zone will directly connect to the Zone II, and the right side of $\mathrm{N}$-th small zone is considered to be impermeable. The boundary conditions could be explicitly presented as follows,

$$
\left.m_{2}\right|_{y=l_{1}}=\left.m_{3,1}\right|_{y=l_{1}},\left.\quad k_{2} e^{-\gamma_{2}\left(m_{0}-m_{2}\right)} \frac{\partial m_{2}}{\partial y}\right|_{y=l_{1}}=\left.k_{3,1} e^{-\gamma_{3,1}\left(m_{0}-m_{3,1}\right)} \frac{\partial m_{3,1}}{\partial y}\right|_{y=l_{1}},\left.\quad \frac{\partial m_{3, N}}{\partial y}\right|_{y=y_{e}}=0
$$

Obtained from Fick's first law of diffusion, the diffusion rate of shale gas can be expressed as:

$$
\frac{\partial V}{\partial t}=-\phi_{3 m} \frac{\partial V_{E}}{\partial t}
$$

Substituting Langmuir isotherm equation Eqs.(2) into Eqs.(21), we can obtain:

$$
\frac{\partial V}{\partial t}=-\phi_{3 m} V_{L} \frac{m_{L}}{\left(m_{L}+m_{3 m}\right)^{2}} \frac{\partial m_{3 m}}{\partial t}
$$

Substituting predefined dimensionless variables to Eq.(17) (22) could obtain their dimensionless formulas, and then aforementioned perturbation transformation proposed by Pedrosa O.A is used to eliminate the non-linearity. Finally, their dimensionless formulas with the consideration of stress sensitivity effects can be as follows

Fracture system:

$$
\frac{\partial^{2} \eta_{3, j D}}{\partial y_{D}^{2}}+\left.\frac{k_{4 D}}{k_{3, j D}} \frac{\partial m_{4 D}}{\partial y_{D}}\right|_{x_{D}=1}+\frac{\lambda}{k_{3, j D}}\left(\eta_{3 m D}-\eta_{3, j D}\right)=\frac{\omega_{3, j D}}{k_{3, j D}} \frac{\partial \eta_{3, j D}}{\partial t_{D}}, l_{j, D}<y_{D}<l_{j+1, D}
$$

Matrix system:

$$
-\lambda\left(\eta_{3 m D}-\eta_{3, j D}\right)=\omega_{3 m D} \frac{\partial \eta_{3 m D}}{\partial t_{D}}+V_{D L} \frac{m_{D L}}{\left(m_{D L}+m_{D i}-m_{3 m D}\right)^{2}} \frac{\partial \eta_{3 m D}}{\partial t_{D}}
$$

With the boundary conditions:

$$
\begin{gathered}
\left.\gamma_{3, j D} \eta_{3, j D}\right|_{x_{D}=l_{j, D}}=\left.\gamma_{3, j+1 D} \eta_{3, j+1 D}\right|_{x_{D}=l_{j, D}},\left.\quad \frac{\partial \eta_{3, j D}}{\partial y_{D}}\right|_{y_{D}=l_{j, D}}=\left.\frac{k_{3, j+1 D}}{k_{3, j D}} \frac{\partial \eta_{3, j+1 D}}{\partial y_{D}}\right|_{y_{D}=l_{j, D}}, j=2, \ldots, N-1 \\
\gamma_{3,1 D} \eta_{3,1 D}=\gamma_{2 D} \eta_{2 D},\left.\quad \frac{\partial \eta_{3,1 D}}{\partial y_{D}}\right|_{y_{D}=l_{1, D}}=\left.\frac{k_{2 D}}{k_{3,1 D}} \frac{\partial \eta_{2 D}}{\partial y_{D}}\right|_{y_{D}=l_{2, D}},\left.\frac{\partial \eta_{3, N D}}{\partial y_{D}}\right|_{y_{D}=l_{N, D}}=0
\end{gathered}
$$

Similarly, Laplace transformation can be used to transform these equations as follows: Fracture system: 


$$
\frac{d^{2} \overline{\eta_{3, j D}}}{d y_{D}{ }^{2}}+\left.\frac{k_{4 D}}{k_{3, j D}} \frac{d \overline{m_{4 D}}}{d y_{D}}\right|_{x_{D}=1}+\frac{\lambda}{k_{3, j D}}\left(\overline{\eta_{3 m D}}-\overline{\eta_{3, j D}}\right)=\frac{\omega_{3, j D}}{k_{3, j D}} s \overline{\eta_{3, j D},} l_{j, D}<y_{D}<l_{j+1, D}
$$

Matrix system:

$$
-\lambda\left(\overline{\eta_{3 m D}}-\overline{\eta_{3, j D}}\right)=\omega_{3 m D} s \overline{\eta_{3 m D}}
$$

With the boundary conditions:

$$
\begin{gathered}
\left.\gamma_{3, j D} \overline{\eta_{3, j D}}\right|_{x_{D}=l_{j, D}}=\left.\gamma_{3, j+1 D} \overline{\eta_{3, j+1 D}}\right|_{x_{D}=l_{j, D}},\left.\quad \frac{d \overline{\eta_{3, j D}}}{d y_{D}}\right|_{y_{D}=l_{j, D}}=\left.\frac{k_{3, j+1 D}}{k_{3, j D}} \frac{d \overline{\eta_{3, j+1 D}}}{d y_{D}}\right|_{y_{D}=l_{j, D}}, j=2, \ldots, N-1 \\
\gamma_{3,1 D} \overline{\eta_{3,1 D}}=\gamma_{2 D} \overline{\eta_{2 D}},\left.\quad \frac{d \overline{\eta_{3,1 D}}}{d y_{D}}\right|_{y_{D}=l_{1, D}}=\left.\frac{k_{2 D}}{k_{3,1 D}} \frac{d \overline{\eta_{2 D}}}{d y_{D}}\right|_{y_{D}=l_{2, D}},\left.\frac{d \overline{\eta_{3, N D}}}{d y_{D}}\right|_{y_{D}=l_{N, D}}=0
\end{gathered}
$$

\subsubsection{Zone IV}

Zone IV is assumed to be an USVR area without any fracturing stimulation as illustrated in Fig.2(a). As we have shown in the previous process of model derivations, Zone IV simultaneously supplies gas to Zone II and Zone III. When the stress sensitivity is considered, the governing function can be presented as follows,

$$
\frac{\partial^{2} m_{4}}{\partial x^{2}}+\gamma_{4}\left(\frac{\partial m_{m_{4}}}{\partial x}\right)^{2}=e^{\gamma_{4}\left(m_{0}-m_{4}\right)} \frac{\phi_{4} \mu C_{g}}{k_{4}} \frac{\partial m_{4}}{\partial t}, x_{f}<x<x_{e}
$$

The upper condition is assumed to be impermeable

$$
\left.\frac{\partial m_{4}}{\partial x}\right|_{x=x_{e}}=0
$$

The lower boundary is adjacent to Zone II and Zone III along with the $y$ direction. Specifically, this lower boundary condition can be presented.

$$
\left.m_{4}\right|_{x=x_{f}}= \begin{cases}\left.m_{2}\right|_{x=x_{f}}, & w_{f} / 2<y<l_{1} \\ \left.m_{3, j}\right|_{x=x_{f}}, & l_{j-1}<y<l_{j}\end{cases}
$$

Their dimensionless formulas with the consideration of stress sensitivity effects can be as follows

$$
\frac{\partial^{2} \eta_{4 D}}{\partial x_{D}^{2}}=\frac{\omega_{4 d}}{k_{4 D}} \frac{\partial \eta_{4 D}}{\partial t_{D}}, 1<x_{D}<x_{e D}
$$

With the upper and lower boundary conditions

$$
\left.\frac{\partial \eta_{4 D}}{\partial x_{D}}\right|_{x_{D}=x_{e D}}=0,\left.\quad \gamma_{4 D} \eta_{4 D}\right|_{x_{D}=1}=\left\{\begin{array}{l}
\left.\gamma_{2 D} \eta_{2 D}\right|_{x_{D}=1}, \quad w_{f D} / 2<y_{D}<l_{1, D} \\
\left.\gamma_{3, j D} \eta_{3, j D}\right|_{x_{D}=1}, \quad l_{j-1, D}<y_{D}<l_{j, D}
\end{array}\right.
$$

Laplace transformation is used to transform these equations Laplace domain

$$
\begin{aligned}
& \frac{d^{2} \overline{\eta_{4 D}}}{d x_{D}^{2}}=s \frac{\omega_{4 d}}{k_{4 D}} \overline{\eta_{4 D}}, 1<x_{D}<x_{e D} \\
& \left.\frac{d \overline{\eta_{4 D}}}{d x_{D}}\right|_{x_{D}=x_{e D}}=0,\left.\gamma_{4 D} \overline{\eta_{4 D}}\right|_{x_{D}=1}= \begin{cases}\left.\gamma_{2 D} \overline{\eta_{2 D}}\right|_{x_{D}=1}, & w_{f D} / 2<y_{D}<l_{1, D} \\
\left.\gamma_{3, j D} \overline{\eta_{3, j D}}\right|_{x_{D}=1}, & l_{j-1, D}<y_{D}<l_{j, D}\end{cases}
\end{aligned}
$$




\subsection{Solution of Multi-Linear Model}

On the basis of solution methods proposed by Ozkan et al, (2011) and Stalgorova et al (2013), this coupled multi-linear models from Eq.3 to Eq.35 can be solved starting the Zone IV. The general solutions of partial differential Eq.34 and Eq.35 can be presented,

$$
\overline{\eta_{4 D}}=A_{4} e^{\sqrt{s \frac{\omega_{4 d}}{k_{4 D}}}} x_{D}+B_{4} e^{-\sqrt{s \omega_{\frac{\omega_{4}}{2}}} x_{D}}
$$

The upper boundary condition can derive the relationship between $A_{4}$ and $B_{4}$,

$$
B_{4}=A_{4} e^{2 \sqrt{s} \frac{\omega_{4 d}}{k_{4 D}}} x_{e D}
$$

Two lower boundary conditions as shown in Eq.(30) could get different solutions

$$
\overline{\eta_{4 D}}= \begin{cases}\frac{\gamma_{2 D} \cosh \left[\sqrt{s \frac{\omega_{4 d}}{k_{4 D}}}\left(x_{D}-x_{e D}\right)\right]}{\gamma_{4 D} \cosh \left[\sqrt{s \frac{\omega_{4 d}}{k_{4 D}}}\left(1-x_{e D}\right)\right]}, \quad w_{f D} / 2<y_{D}<l_{1, D} \\ \frac{\gamma_{3 j D} \cosh \left[\sqrt{\frac{\omega_{4 d}}{k_{4 D}}}\left(x_{D}-x_{e D}\right)\right]}{\gamma_{4 D} \cosh \left[\sqrt{\frac{\omega_{4 d}}{k_{4 D}}}\left(1-x_{e D}\right)\right]} \overline{\eta_{3 j D}}, \quad l_{j-1, D}<y_{D}<l_{j, D}\end{cases}
$$

After obtaining the solutions in Zone IV, the equations for Zone II and Zone III should be coupled together to simultaneously obtain the analytical solutions. After substituting Eq.(38) to Eq.(15) and Eq.(26), the effects of Zone IV can be incorporated into Zone II and Zone III.

Zone II: $\frac{d^{2} \overline{\eta_{2 D}}}{d y_{D}^{2}}=\left\{\frac{\gamma_{2 D} k_{4 D}}{\gamma_{4 D} k_{2 D}} \sqrt{s \frac{\omega_{4 D}}{k_{4 D}}} \tanh \left[\sqrt{s \frac{\omega_{4 d}}{k_{4 D}}}\left(1-x_{e D}\right)\right]+s \frac{\omega_{2 D}}{k_{2 D}}\right\} \overline{\eta_{2 D}}, w_{f D} / 2<y_{D}<l_{1, D}$

\section{Zone III:}

$$
\frac{d^{2} \overline{\eta_{3, j D}}}{d y_{D}^{2}}=\left\{\frac{\gamma_{3 j D} k_{4 D}}{\gamma_{4 D} k_{3, j D}} \sqrt{s \frac{\omega_{4 D}}{k_{4 D}}} \tanh \left[\sqrt{s \frac{\omega_{4 d}}{k_{4 D}}}\left(1-x_{e D}\right)\right]+\frac{\lambda \omega_{3 m D} s}{\left(\lambda+\omega_{3 m D} s\right) k_{3, j D}}+s \frac{\omega_{3, j D}}{k_{3, j D}}\right\} \overline{\eta_{3, j D}}, l_{j, D}<y_{D}<l_{j+1, D}
$$

To simplify the notation,

$$
\alpha_{j}=\sqrt{\frac{\gamma_{3 j D} k_{4 D}}{\gamma_{4 D} k_{2, j D}} \sqrt{\frac{\gamma_{2 D} k_{4 D}}{\gamma_{2 D} k_{3 D}} \sqrt{\frac{\omega_{4 D}}{k_{4 D}}}} \tanh \left[\sqrt{s \frac{\omega_{4 D}}{k_{4 D}}}\left(1-x_{e D}\right)\right]+\frac{\lambda \omega_{3 m D} s}{\left(\lambda+\omega_{3 m D} s\right) k_{3, j D}}+s \frac{\omega_{3, j D}}{k_{3, j D}}}, j=1,2, \ldots, N
$$

Similarly, the general solutions for Eq.(39) and Eq.(40) can be described as follow,

\section{Zone II:}

$$
\overline{\eta_{2 D}}=A_{2} e^{\beta x_{D}}+B_{2} e^{-\beta x_{D}}, w_{f D} / 2<y_{D}<l_{1, D}
$$

\section{Zone III:}

$$
\overline{\eta_{3, j D}}=A_{3, j} e^{\alpha_{j} x_{D}}+B_{3, j} e^{-\alpha_{j} x_{D}}, l_{j, D}<y_{D}<l_{j+1, D}
$$


There are in total $2(N+1)$ coefficients needed to be determined. That is to say, we should write $2(N+1)$ equations to obtain an unique solutions. Specifically, there are $N$ interfaces in the coupled area of Zone II and Zone III which allows us to write $2 N$ equations.

At the interface of neighboring small sub-sections in Zone III :

$$
\gamma_{3, j D}\left(A_{3, j} e^{\alpha_{j} l_{j, D}}+B_{3, j} e^{-\alpha_{j} l_{j, D}}\right)=\gamma_{3, j+1 D}\left(A_{3, j+1} e^{\alpha_{j+1} l_{j, D}}+B_{3, j+1} e^{-\alpha_{j+1} l_{j, D}}\right)
$$

and

$$
k_{3, j D}\left(\alpha_{j} A_{3, j} e^{\alpha_{j} l_{j, D}}-\alpha_{j} B_{3, j} e^{-\alpha_{j} l_{j, D}}\right)=k_{3, j+1 D}\left(\alpha_{j+1} A_{3, j+1} e^{\alpha_{j+1} l_{j, D}}-\alpha_{j+1} B_{3, j+1} e^{-\alpha_{j+1} l_{j, D}}\right)
$$

At the interface of Zone II and Zone III:

$$
\gamma_{3,1 D}\left(A_{3,1} e^{\alpha_{1} l_{1, D}}+B_{3,1} e^{-\alpha_{1} l_{1, D}}\right)=\gamma_{2 D}\left(A_{2} e^{\beta l_{1, D}}+B_{2} e^{-\beta l_{1, D}}\right)
$$

and

$$
k_{3,1 D}\left(\alpha_{1} A_{3,1} e^{\alpha_{1} l_{1, D}}-\alpha_{1} B_{3,1} e^{-\alpha_{1} l_{1, D}}\right)=k_{2 D}\left(\beta A_{2} e^{\beta l_{1, D}}-\beta B_{2} e^{-\beta l_{1, D}}\right)
$$

Other two equations can be further obtained at the inner boundary of Zone II and outer boundary of Zone III.

$$
\gamma_{1 D} \overline{\eta_{1 D}}=\gamma_{2 D}\left(A_{2} e^{\alpha_{1} \frac{w_{f D}}{2}}+B_{2} e^{-\alpha_{1} \frac{w_{f D}}{2}}\right)
$$

and

$$
A_{3, N} e^{\beta y_{e D}}-B_{3, N} e^{-\beta y_{e D}}=0
$$

All these equations can be used to determine the required coefficients. After obtaining the solution at Zone II, we substitute this equation into Eqs.(11) as follows

$$
\frac{d^{2} \overline{\eta_{1 D}}}{d x_{D}^{2}}=\left[s \frac{\omega_{1 D}}{k_{1 D}}-\frac{2 k_{2 D}}{C_{1 D}} \beta\left(A_{2} e^{\beta \frac{w_{f D}}{2}}-B_{2} e^{-\beta \frac{w_{f D}}{2}}\right)\right] \overline{\eta_{1 D}}
$$

The general solutions should be

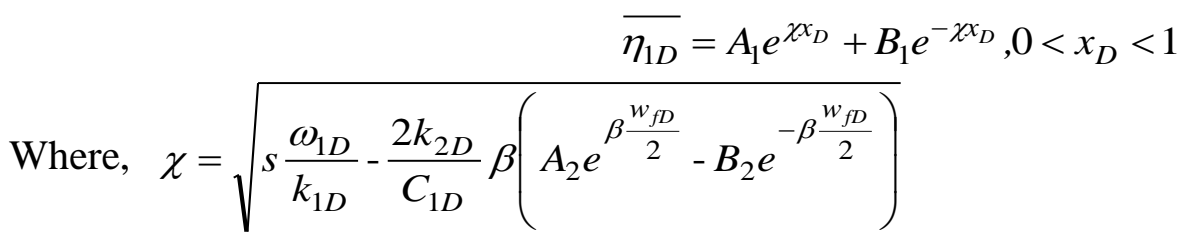
follows

Conditioning to the inner and outer boundary conditions, these two coefficients are as

$$
A_{1}=\frac{\pi}{s C_{1 D} \chi\left(e^{2 \chi}-1\right)}, B_{1}=\frac{\pi e^{2 \chi}}{s C_{1 D} \chi\left(e^{2 \chi}-1\right)}
$$

Finally, we can obtain the botton-hole pressure

$$
\overline{\eta_{w D}}=\left.\overline{\eta_{1 D}}\right|_{x_{D}=0}=\frac{\pi\left(e^{2 \chi}+1\right)}{s C_{1 D} \chi\left(e^{2 \chi}-1\right)}
$$

Following the methods used in Xu et al. (2013) the zero-order perturbation solution of the bottom-hole pressure in the Laplace space considering the wellbore storage $C_{D}$ and the skin factor $S$ is obtained: 


$$
\bar{\eta}_{w D}\left(S, C_{D}\right)=\frac{s \bar{\eta}_{w D}+S}{s+C_{D} s^{2}\left[s \bar{\eta}_{w D}+S\right]}
$$

Finally, by using Stefest numerical inversion methods proposed by Stefest (1970), the zero-order perturbation solution of the bottom hole pressure in real space is obtained, and the real bottom hole pressure $m_{w D}$ can be obtained by Eq. (6).

\section{Model verification}

As mentioned above, previous models have not taken the stress sensitivity and the SRV into consideration, simultaneously, some simplifications need to be imposed in order to validate our model with the previous ones. Wu et al. (2019) have established a pressure analysis model for multi-fractured horizontal wells in homogeneous and stress-sensitive reservoirs. Further analysis of the proposed model in this work reveals that if we do not subdivide the inner SRV subsections, our model can be simplified to be a four-linear flow model and the solution for multi-fractured horizontal wells in homogeneous and stresssensitive reservoirs can be obtained. To verify the correctness of our model, comparison is made with the model proposed by $\mathrm{Wu}$ et al. (2019). The case is a fractured horizontal well with 5 fractures perpendicular to wellbore in stress-sensitive tight gas reservoirs as shown in Figure 1. The other parameters used for simulation are listed in Table 1. As shown in Figure 2 , there is a perfect agreement between the results of the two models indicates the validation of our model.

Table 1: Basic data used for model

\begin{tabular}{lll}
\hline Parameters & Value & Units \\
\hline Reference length, $L_{\text {ref }}$ & 0.1 & $\mathrm{~m}$ \\
Hydraulic fracture permeability in zone I, $k_{1}$ & 1000 & $\mathrm{mD}$ \\
Permeability in SRV zone II, $k_{2}$ & 0.1 & $\mathrm{mD}$ \\
Permeability in SRV zone III, $k_{3}$ & 0.01 & $\mathrm{mD}$ \\
Permeability in zone IV, $k_{4}$ & 0.001 & $\mathrm{mD}$ \\
Initial formation pressure,,$p_{\mathrm{i}}$ & 30 & $\mathrm{MPa}$ \\
Fluid viscosity & $2.7 \times 10^{-3}$ & $\mathrm{mpa}^{-5}$ \\
Compressibility in zone I, $C t_{1}$ & $5 \times 10^{-4}$ & $\mathrm{MPa}^{-1}$ \\
Compressibility in SRV zone II, $C t_{2}$ & $5 \times 10^{-4}$ & $\mathrm{MPa}^{-1}$ \\
Compressibility in SRV zone III, $C t_{3}$ & $5 \times 10^{-4}$ & $\mathrm{MPa}^{-1}$ \\
Compressibility in zone IV, $C t_{4}$ & $5 \times 10^{-4}$ & $\mathrm{MPa}^{-1}$ \\
Porosity in zone I & 0.15 & fraction \\
Porosity in SRV zone II & 0.15 & fraction \\
Porosity in SRV zone III & 0.15 & fraction \\
Porosity in zone IV & 0.15 & fraction \\
Half length of the fractures, $x_{\mathrm{f}}$ & 100 & $\mathrm{~m}$ \\
Width of the SRV zone, $y_{\mathrm{e}}$ & 100 & $\mathrm{~m}$ \\
Length of the SRV zone, $x_{\mathrm{e}}$ & 150 & $\mathrm{~m}$
\end{tabular}




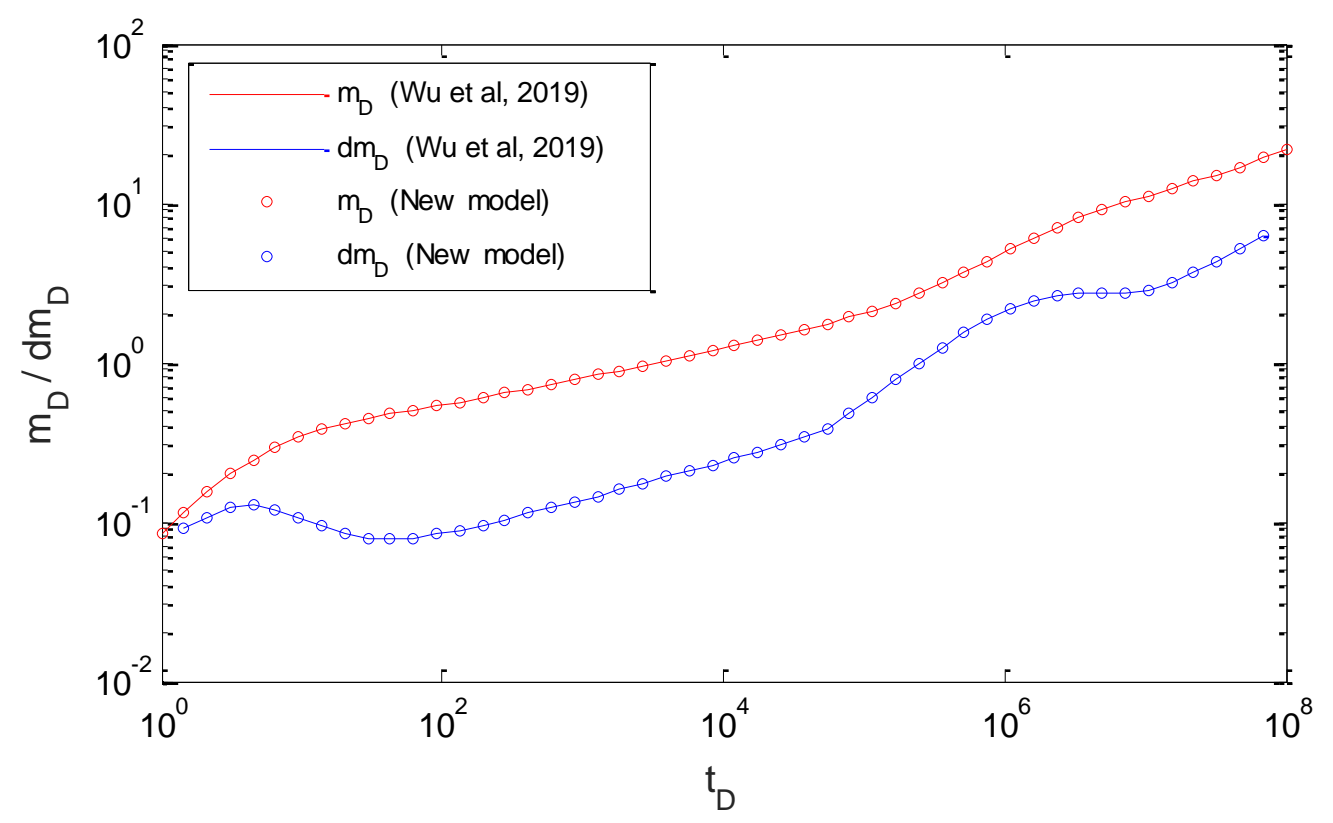

Figure 2: Comparison between the model in this paper and that in 0

In addition, our new model has the ability to subdivide the inner SRV zones into small subsections with different permeability. Our model can be equivalent to standard four-linear flow models if all subsections have the same permeability. In numerical experiment, we subdivide the SRV zones III into 1 (without division), 5, 20 subsections. Figure 3 shows the the evolution of dimensionless pressure and its derivative curves. The number of subsections has no any influences on the pressure curves, Which indicates that our proposed models can be generalized to be any multi-linear flow models.

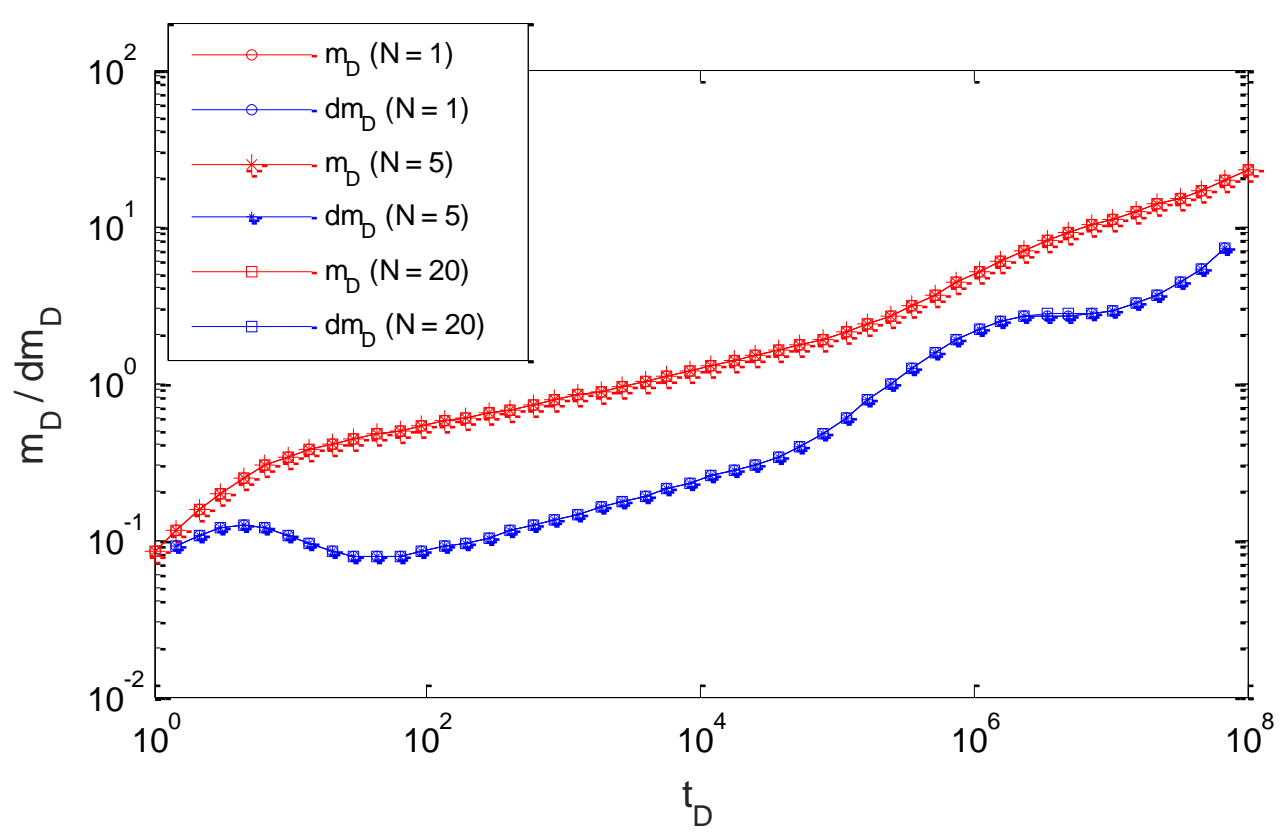

Figure3: Investigation of number of subdivision of SRV zones III on the type curves 


\section{Results and discussions}

\subsection{Transient pressure behaviors analysis}

Typical pressure response curves of a multi-fractured horizontal well with the consideration of SRV zone and the stress sensitivity are shown in Figure 4. The fracture distribution and geometry of the SRV zone and USRV zone also can be seen in Figure 2. We take the reference length $L_{\text {ref }}$ as $0.1 \mathrm{~m}$. The values of the relevant dimensionless variables used in this numerical experiments are as follows: $\quad C_{f D}=10, \quad k_{2 D}=100, \quad k_{3 D}=10, \quad k_{4 D}=1, \quad \omega_{3 D}=0.01, \quad \lambda=0.15, \quad m_{D L}=0.12, \quad V_{D L}=0.1, \quad$, $\gamma_{D}=0.05, C_{D}=0.001, S=0.1$. By analyzing the $\log$ - log graphs of dimensionless wellbore hole pressure $\left(p_{w D}\right)$ and its derivative $\left(\mathrm{d} p_{w D} / \mathrm{d} \ln \left(t_{D} / C_{D}\right)\right)$ vs. dimensionless times, we find that approximately five flow stages can be identified.

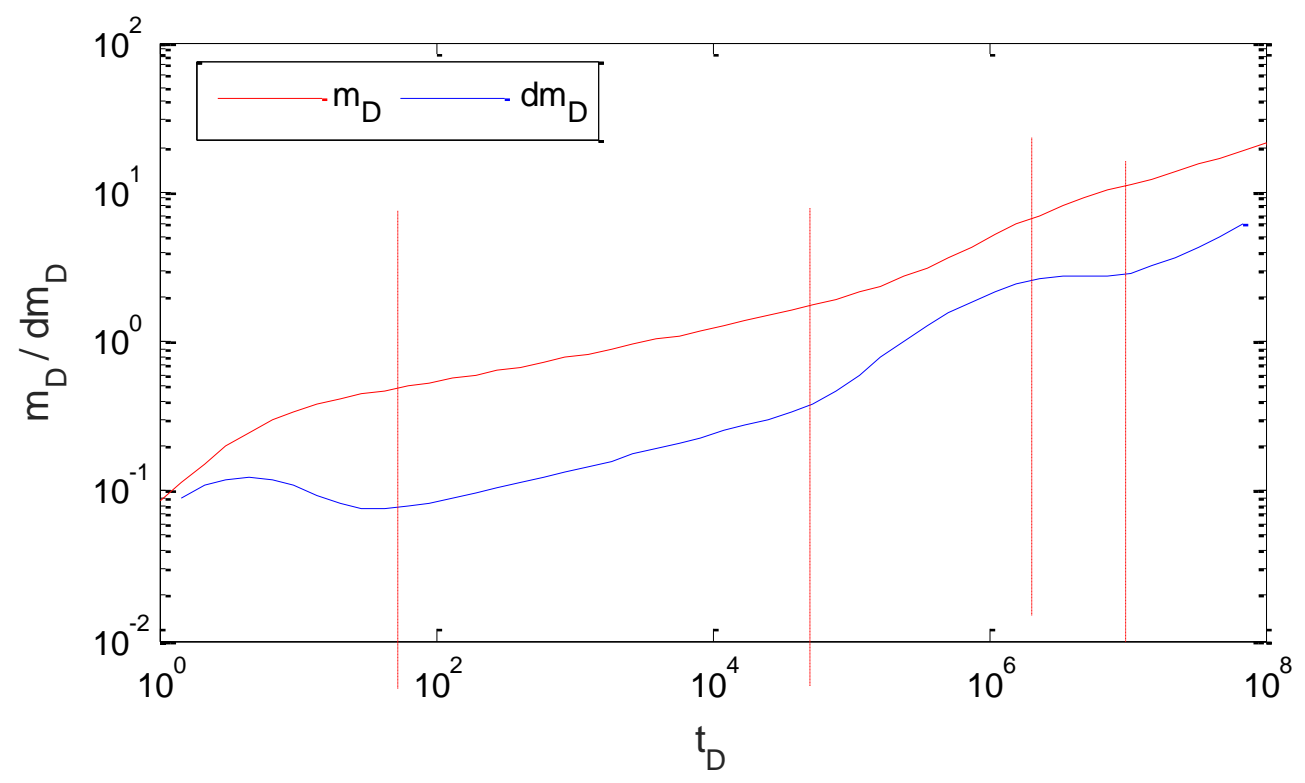

Figure 4: Typical curves of pressure response for multi-fractured-fractured horizontal well in stress-sensitivity SGR formation

Stage I: Wellbore storage and skin effect flow. This is a relatively common flow stage. The wellbore storage coefficient and the skin representing the flow choking between the hydraulic fractures and the wellbore can be obtained by analyzing the characteristic of pressure and pressure derivative cures in this stage. The detailed explanation of this stage can be found in the literature (Agarwal et al, 1970; Kuchuk et al, 2010; Song et al, 2011).

Stage II: The formation bin-linear flow in the inner SRV. In this flow regime, due to the high permeability of the hydraulic fracture networks, hydraulic fractures can be treated as linear sinks, liquid enters the hydraulic fractures in the directions perpendicular to the fracture faces. The typical feature of this flow behavior is that a 1/4- slope straight line (Figure 4) occurs on the dimensionless derivative pressure curve. We also should note that due to the high-permeability of hydraulic fractures, the fracture linear flow is also blurred in this stage. This phenomenon is also consistent with the observations from the realistic applications.

Stage III: The formation linear flow in the SRV zone and the transition flow between SRV zone II and zone IV. In this stage, the typical feature of this flow behavior is that a 1/2- 
slope straight line (Figure 4) occurs on the dimensionless derivative pressure curve. In addition, the fluid in the zone IV also starts to supply the SRV zone. The fluid supply of the zone IV slows down the pressure depletion evidently. The typical characteristic is there is a "recess" on the pressure derivative curves.

Stage IV: the transition flow between SRV zone II and zone III. The stress sensitivity begins to take effects in this regime, the pressure wave reaches the boundary of the SRV. However, due to the lower permeability of the unstimulated region, there is no enough fluid supply in the outer region. The flow characteristic in this stage is essential reflective of boundary-dominated flow. This is mainly because the pressure interference between the fracture and the property difference between the SRV and the outer region. The fracture and boundary of the SRV dominates the pressure responses. The similar flow stage is also discussed by Ren et al. (2016, 2017), when they studied transient pressure behaviors of a volume fractured horizontal well with a discrete fracture network.

Stage V: The formation linear flow in zone III. As we have idealized the outer unstimulated region as single porosity media to ignore the pre-existing natural fractures in the tight oil reservoirs. When the pressure waves reach the outer region, there is fluids feed from the matrix to the natural fracture in the outer region. In this stage, the typical feature of this flow behavior is that a 1/2- slope straight line (Figure 4) occurs on the dimensionless derivative pressure curve 0 . The effects of stress sensitivity on the transient pressure response become evident in this stage, which will be systematically analyzed in the following part.

It should be noted the characteristics of the above flow stages can be affected by various factors, such as the physical properties of the SRV and the out region, the stress sensitivity, the geometry of the SRV zone. In practice, not all flow periods would appear on type curves in a single situation. Therefore, effects of key variables on the typical curves need to be further studied.

\subsection{Sensitivity analysis}

To systematically investigate the transient pressure behaviors of the multi-fractured horizontal well in stress sensitive SGR by use of our proposed multi-linear flow model, sensitivity analysis is conducted in this section to analyse the effect of critical parameters on the typical pressure response curves.

\subsubsection{Effect of stress sensitivity}




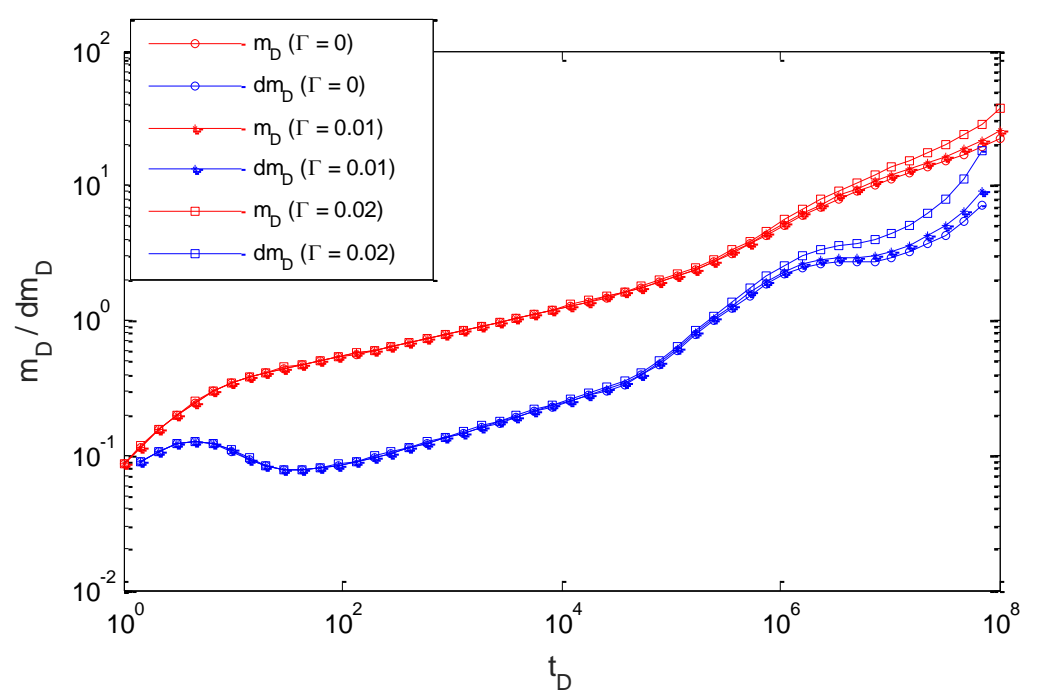

(a)

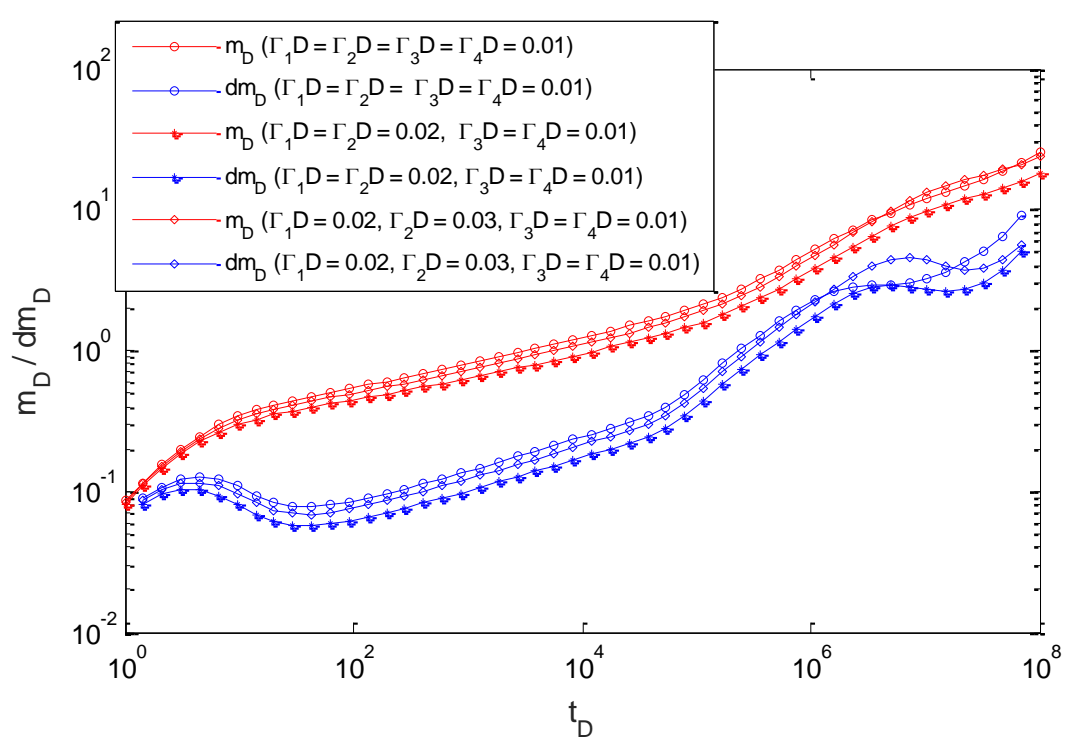

(b)

Figure 5: The effect of permeability modulus on pressure and derivative curves, (a) constant permeability module for all zones; (b) each zone has different permeability module

Figure 5 shows the effect of stress sensitivity on the pressure transient curves. The values of relevant parameters are listed as follows: $C_{f D}=10, k_{2 D}=100, k_{3 D}=10, k_{4 D}=1, \omega_{3 D}=0.01, \lambda=0.15, m_{D L}=0.12, V_{D L}=0.1, C_{D}=0.001, S=0.1$. This newly proposed multi-linear flow model can be used to analyze two situations: (a) there is a constant permeability module for all zones; (b) each zone has different permeability module. We will separately investigate these two situations.

When we make an assumption that there is a constant permeability module for all zones, three cases were studied in which the dimensionless permeability modulus $\gamma_{D}$ is equal to 0 , 0.01 and 0.02, respectively as illustrated in Figure 5(a). It can be seen from Figure 5(a) that as the dimensionless permeability modulus increases, the dimensionless pressure and its derivative curves rise gradually, the stress sensitivity mainly affect the flow behaviors in intermediate and later flow stages. In stress sensitive shale gas reservoirs, as the fluid is produced, the gradual reduction of formation pressure will result in a decrease of the 
permeability of the system and a growing of pressure depletion. When the dimensionless permeability modulus increases to a certain value, the pressure derivative curve rises up significantly in later periods, showing the characteristic of closed boundary.

When we assume that each zone has different permeability module, three cases also were studied as illustrated in Figure 5(a). As we can see from Figure 5(b), the stress sensitivity will have significant influences on the entire flow stages, which is significantly different from the previous situation. This finding is very important for us to enhance the gas recovery from shale gas reservoir. we need to design reasonable production scheme to maintain the formation pressure. Both zone I and SRV zone II are closed to the wellbore, as a result, the pressure drops are significant and hence causes severe stress sensitivity. Thus, larger stress sensitivity coefficients in SRV zone generally induce larger pressure drops.

\subsubsection{Effect of the mobility capacity of the zone I and SRV zone II}

Transient pressure and pressure derivative curves for different mobility capacity of the zone I and SRV zone II are illustrated in Figure 6. The values of relevant parameters are listed as follows:

$k_{3 D}=10, \quad k_{4 D}=1, \quad \omega_{3 D}=0.01, \lambda=0.15, m_{D L}=0.12, \quad V_{D L}=0.1, \gamma_{D}=0.05, C_{D}=0.001, S=0.1$. Вy analyzin, the mobility capacity of the zone I and SRV zone II are represented by dimensionless fracture conductivity $\mathrm{C}_{\mathrm{fD}}$ and dimensionless formation permeability $k_{2 \mathrm{D}}$. In this numerical experiment, the SRV zone III is not divided into subsections, e.g., $\mathrm{N}=1$. It can be seen from Figure 6(a) that dimensionless fracture conductivity $\mathrm{C}_{\mathrm{fD}}$ has significant influences on the flow behaviors of the earl time, especially the wellbore storage and skin effect flow. Other flow stages are almost not affected. This result is consistent with our previous observations that fracture linear flow is easily blurred by the wellbore storage and skin effect flow stage due to its high mobility capacity. Specifically, as $\mathrm{C}_{\mathrm{fD}}$ decreases, the duration of wellbore storage and skin effect flow period will be shortened and the starting time of formation bi-linear flow period in the SRV zone II will be advanced. It is mainly because the mobility capacity determines flow capacity contrast of the SRV and hydraulic fracture. Therefore, the pressure and derivatives responses associated SRV zone will surely enlarge; the curves of the transient response will rise in wellbore storage and skin effect flow.

The mobility capacity $k_{2 \mathrm{D}}$ determines gas flow capacity in the SRV zone II. We set mobility capacity $k_{2 \mathrm{D}}$ separately to be 100,200 and 300 to investigate its effects on the pressure curves. It can be seen from Figure 6(b) that the dimensionless formation permeability $k_{2 \mathrm{D}}$ almost influences the entire flow stages. Specifically, the flow regimes of the shape of type curves are not distorted, the pressure only raise up as the the dimensionless formation permeability $k_{2 \mathrm{D}}$ decreases. This means that a large value of $k_{2 \mathrm{D}}$ increases the gas flow capacity in SRV zone and therefore leads to a small pressure drop. An effective maintenance of formation pressure is very significant to yield a long-term gas production from shale gas reservoir. In addition, we also find a very interesting phenomenon that a "concave" will occur at the transition flow stage between SRV zone II and zone III as the $k_{2 \mathrm{D}}$ increases. Our proposed multi-linear flow model will show a special flow stage for the dualporosity model. The occurrence of a "concave" also can be an indicator that the permeability of induced SRV zone is much larger than that of outer-zone. 


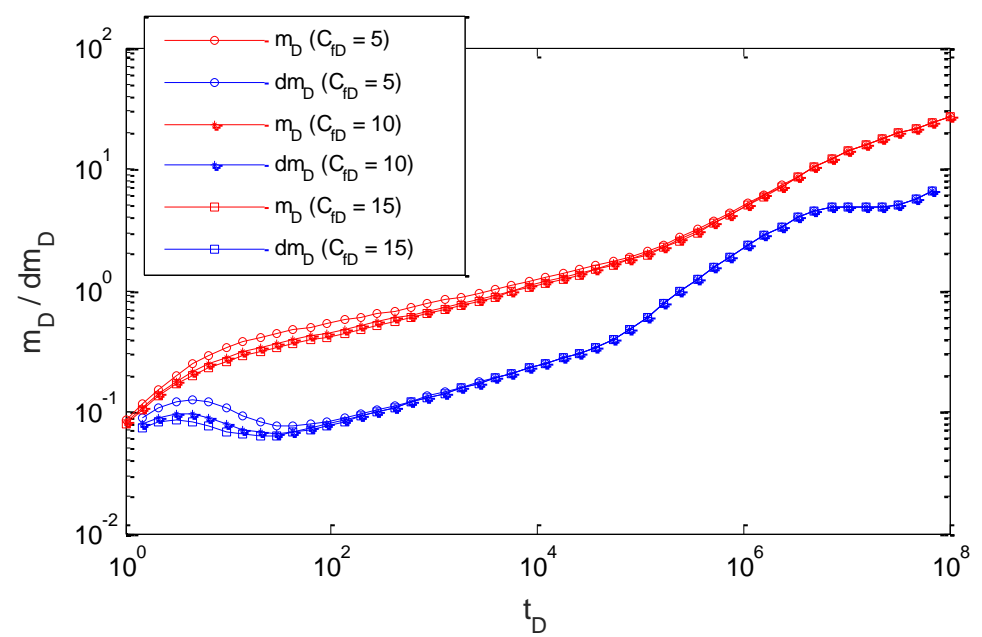

(a) zone I

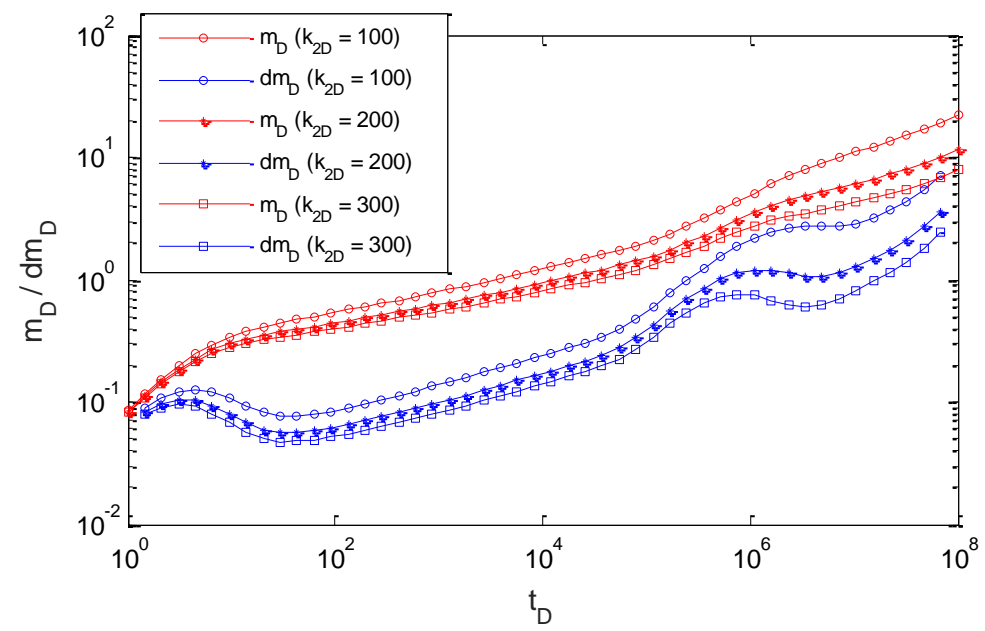

(b) SRV zone II

Figure 6: The effect of mobility capacity of zone I and the SRV zone II on pressure and derivative curves

\subsubsection{Effect of the mobility capacity of the zone III and zone IV}

Transient pressure and pressure derivative curves for different mobility capacity of the SRV zone III and the outer region zone IV are illustrated in Figure 7. The values of relevant parameters are listed as follows: $C_{f D}=10, k_{2 D}=100, \omega_{3 D}=0.01, \lambda=0.15, m_{D L}=0.12, V_{D L}=0.1$, $\gamma_{D}=0.05, C_{D}=0.001, S=0.1$. In this numerical experiment, the SRV zone III is not divided into subsections as wells, e.g., $\mathrm{N}=1$. We can observe from Figure 7(a) that the mobility capacity of the zone III almost has no any influences on the flow behaviors of the early and intermediate flow stages, on the contrary, the transition flow and formation linear flow regimes are severely dominated. This results are also in agreement with our common understanding. Specifically, as the mobility capacity of zone III $k_{3 D}$ decreases, the duration of transition flow period will be enlarged and the starting time of formation linear flow period and boundary-dominated flow stage in the SRV zone III will be delayed.

The mobility capacity $k_{4 \mathrm{D}}$ determines gas flow capacity in the outer zone $\mathbf{I V}$. We set mobility capacity $k_{4 \mathrm{D}}$ separately to be 10,20 and 30 to investigate its effects on the pressure curves. Figure 7(b) apparently demonstrates that the dimensionless formation permeability $k_{4 D}$ has great influence on the formation bi-linear flow stage in SRV zone II. This is very different from the effects of mobility capacity $k_{3 D}$ in SRV zone III. In the process of model development, we assume that the outer zone IV simultaneously supplies gas to SRV zone II 
and SRV zone III. Because SRV zone II is adjacent to the wellbore and thus yields larger pressure drop, the outer zone IV is more prone to supply gas to SRV zone II, as illustrated in Figure 7(b). As the mobility capacity $k_{4 \mathrm{D}}$ decreases, the gas supply will be reduced as well, as a result, the dimensionless pressure curves will be move upward.

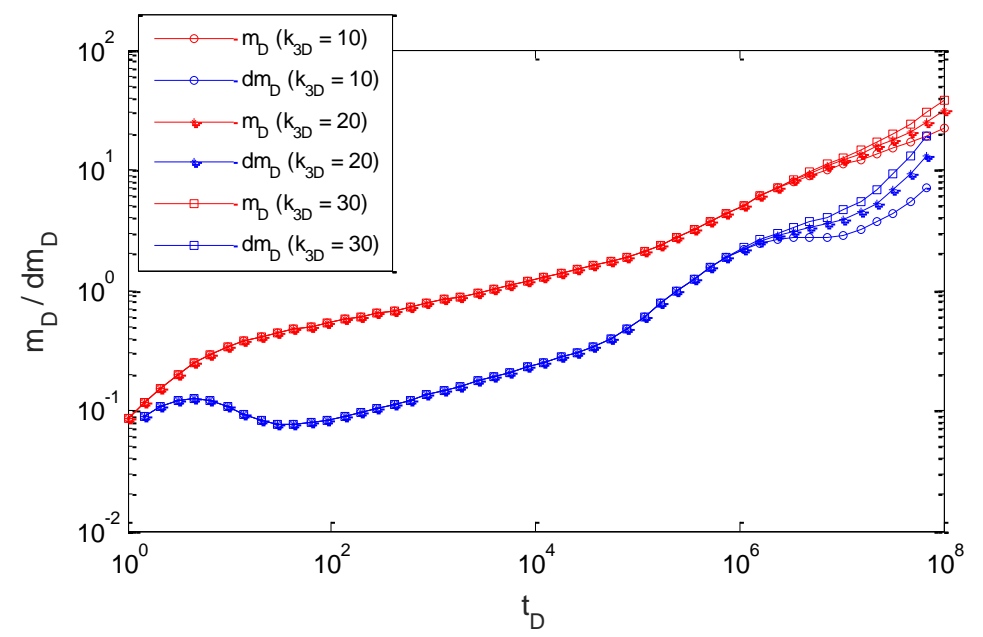

(a) zone III

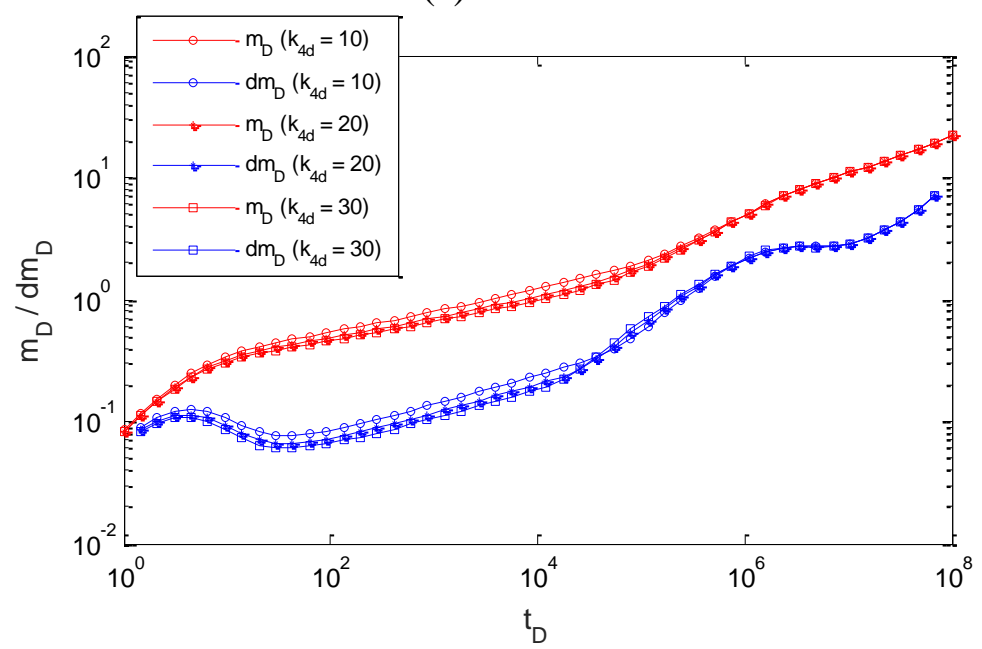

(b) zone IV

Figure 7: The effect of mobility capacity of the zone III and zone IV on pressure and derivative curves

\subsubsection{Effect of the Size of Outer Region}

Three cases with different size of outer regions shown in Figure 8 are studied in this section to identify the effect of size of outer regions on the transient behaviors. The values of relevant parameters are listed follows $C_{f D}=10, \quad k_{2 D}=100, \quad k_{3 D}=10, \quad k_{4 D}=1, \quad \omega_{3 D}=0.01, \quad \lambda=0.15, \quad m_{D L}=0.12, \quad V_{D L}=0.1, \quad$, $\gamma_{D}=0.05, C_{D}=0.001, S=0.1$. As shown in Figure 8, the width and length of outer-region have mainly affected different flow regimes in SRV zone II and SRV zone III. Specifically speaking, the width of outer-region mainly has influences on the formation linear flow regime in SRV zone III, while the width of outer-region mainly impacts the formation bi- 
linear and transition flow regimes. As the size of outer-region decreases, the boundary conditions will be interfere the flow regimes in SRV zone in advance, as a result, the dimensionless pressure and its derivatives will be upper. This phenomenon is very similar to the effects of the mobility capacity of the SRV zone III and zone IV. Enlarging the size of outer-region can decrease the negative effects of boundary condition and increasing the mobility capacity of the zone III and zone IV can decrease the flow resistance in SRV zone, which is beneficial to obtain high production rate.

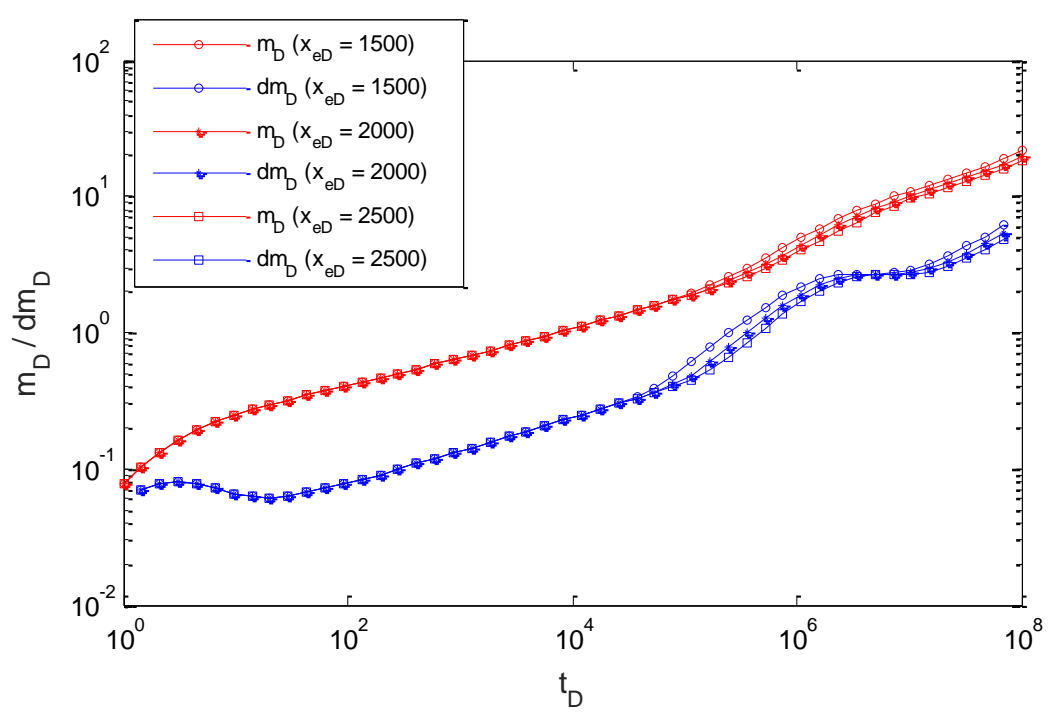

(a) Length of outer region

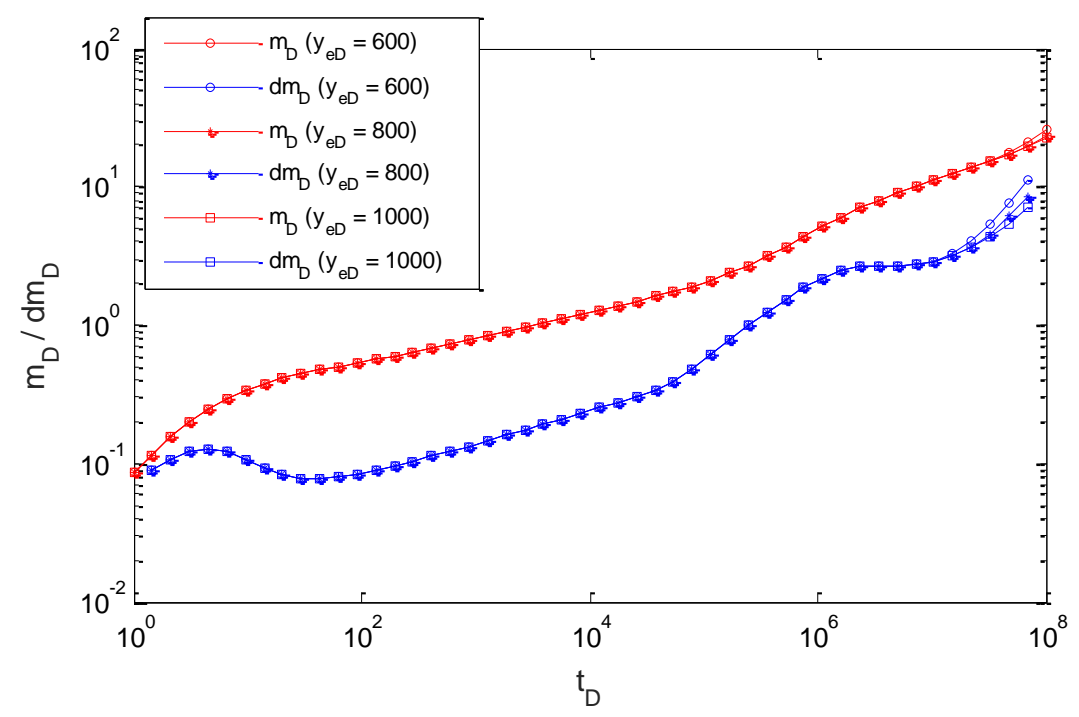

(b) Width of outer region

Figure8: The effect of size of the outer region on pressure and derivative curves

\subsubsection{Effect of the size of SRV zone II}




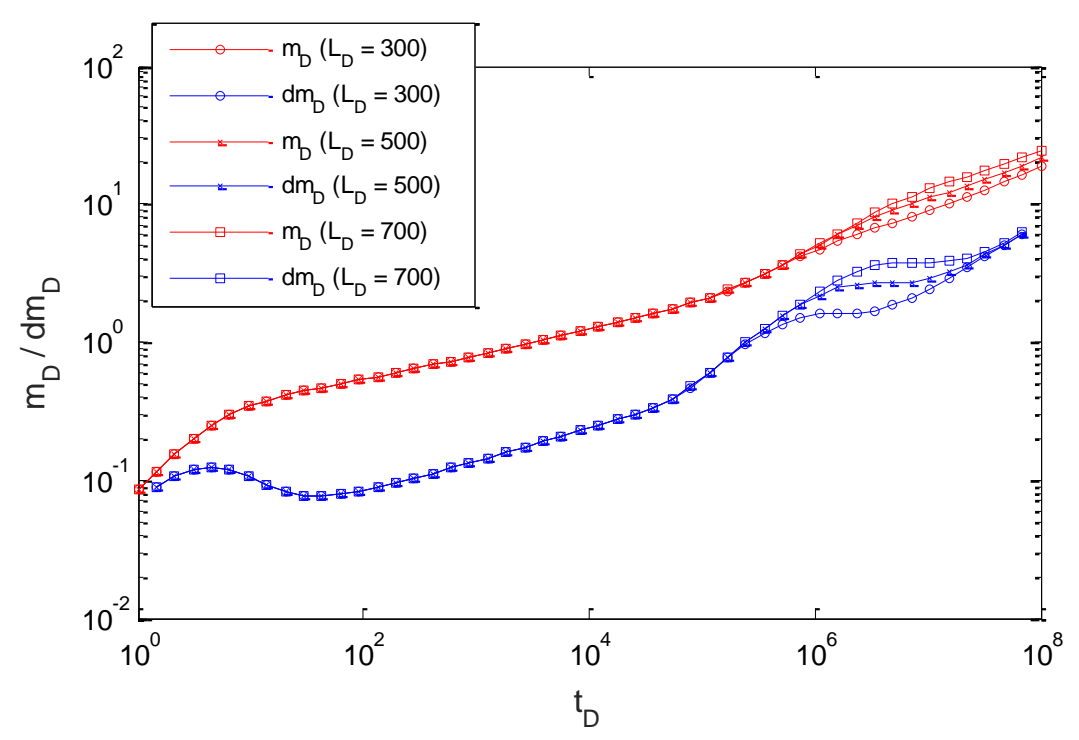

Figure9: The Effect of the size of SRV zone II on pressure and derivative curves

Figure 9 illustrates the effect of the size of SRV zone II on the transient behaviors. The values of relevant parameters are listed as follows: $C_{f D}=10, \quad k_{2 D}=100, \quad k_{3 D}=10, \quad k_{4 D}=1, \quad \omega_{3 D}=0.01, \quad \lambda=0.15, \quad m_{D L}=0.12, \quad V_{D L}=0.1, \quad$, $\gamma_{D}=0.05, C_{D}=0.001, S=0.1$, and the dimensionless size of SRV zone II $L_{D}$ is $300,500,700$, respectively (Figure 9). It can be seen from Figure 9 that the the dimensionless size of SRV zone II can affect all the flow stages after the formation bi-linear flow regimes. The larger size of SRV zone II has (1) a later end of formation bi-linear in the SRV zone II, (2) a postponed beginning of formation linear flow in SRV zone III, (3) the lower values of dimensionless pressure and its derivatives in transition flow regime between SRV zone II and SRV zone III. All these phenomena might provide useful information to identify the sizes of SRV after massive hydraulic fracturing. Figure 9 also shows that the larger the SRV size, the smaller the dimensionless pressure and its derivatives. Smaller dimensionless pressure indicates lower pressure depletion in the formation, which is beneficial to obtain a high production rate. In this paper, Zone II is a highly permeable SVR zone which is adjacent to the main hydraulic fracture as illustrated in Fig.2(a). Zone II is the gas flow path from SGR to wellbore. Increasing the size of this zone will delay the negative effects of outer boundary, which is beneficial to obtain high production rate as well.

\subsubsection{Effect of coefficient of permeability variation}

In this paper, to characterize spatially varying permeability due to the non-uniform distribution of proppant within SRV Zone III, we further divide Zone III into $N$ small zones as illustrated in Fig.1(b). Figure 10 show the effect of coefficient of permeability variation in SRV zone III on the transient behavior, the values of the parameters related to the Figure 10 are listed as follows: $C_{f D}=10, \quad k_{2 D}=100, \quad k_{3 D}=10, \quad k_{4 D}=1, \quad \omega_{3 D}=0.01, \quad \lambda=0.15, \quad m_{D L}=0.12, \quad V_{D L}=0.1, \quad$, $\gamma_{D}=0.05, C_{D}=0.001, S=0.1$. 
To systematically represent the spatial variation of permeability in Zone III, two mathematical formulas, including linear and logarithmic functions, are used to describe the reduction of permeability away from the main hydraulic fracture. Figure 10(a) and Figure 10(b) show the dimensionless permeability for each sub-sections. Figure 10(c) and Figure 10(d) depict the dimensionless permeability as a function of distance to the main hydraulic fracture. In contrast to the linear function, logarithmic function yields a more rapid reduction of dimensionless permeability. What's more, increasing the number of subsections can generate a continuous reduction of permeability.

Figure 10(e) and Figure 10(f) show the effect of coefficient of permeability variation in SRV zone III on the transient behavior. Because the linear function generates a continuous reduction of permeability, large parts of the SRV zone III still preserves relatively high permeability, and only a small part of regions which is adjacent to the outer-boundary will get small permeability. Under this condition, the formation linear flow regime will be mainly impacted as Figure 10(e), as the number of subsections increases, the reduction rate of permeability will be decreases, as a result, the negative of boundary condition will be delayed. On the contrary, the logarithmic function easily leads to a rapid reduction of permeability as illustrated in Figure 10(d). Almost the entire flow regimes are severely influenced. This phenomenon can be considered to be reliable indicator to judge whether the variation of permeability follows a logarithmic function or not. In the real-field applications, a linear variation of permeability induced by massive hydraulic fracturing operation has the potential to obtain high production rate.

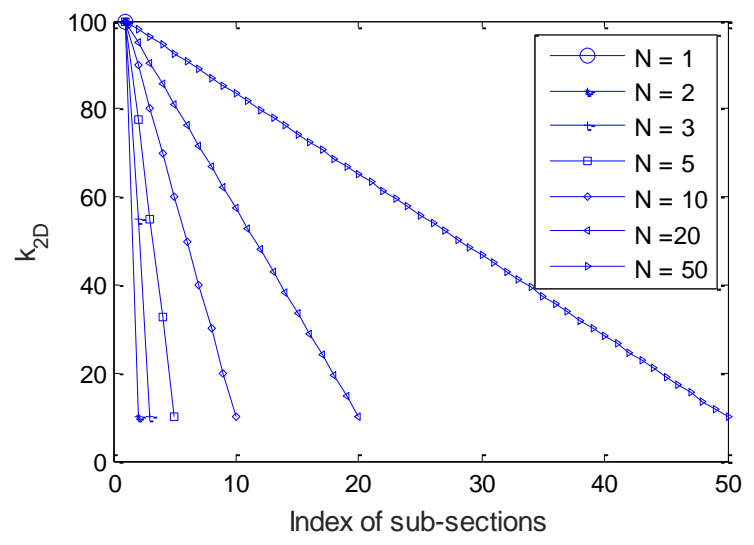

(a) linear function

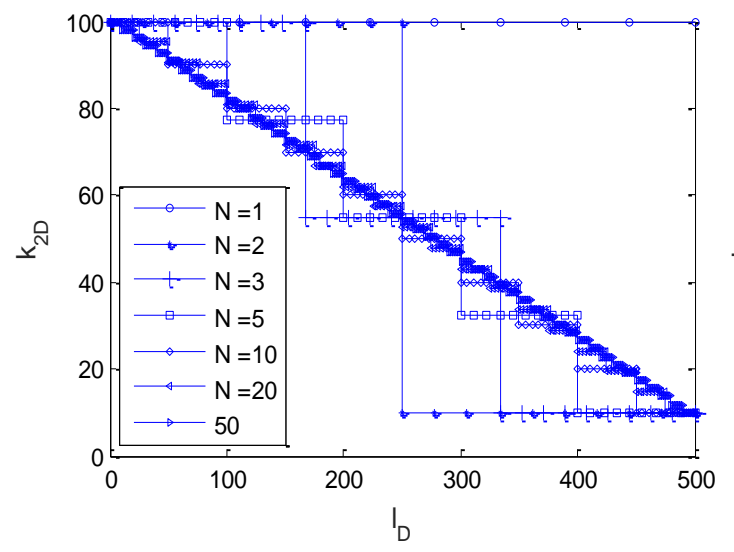

(c) linear function

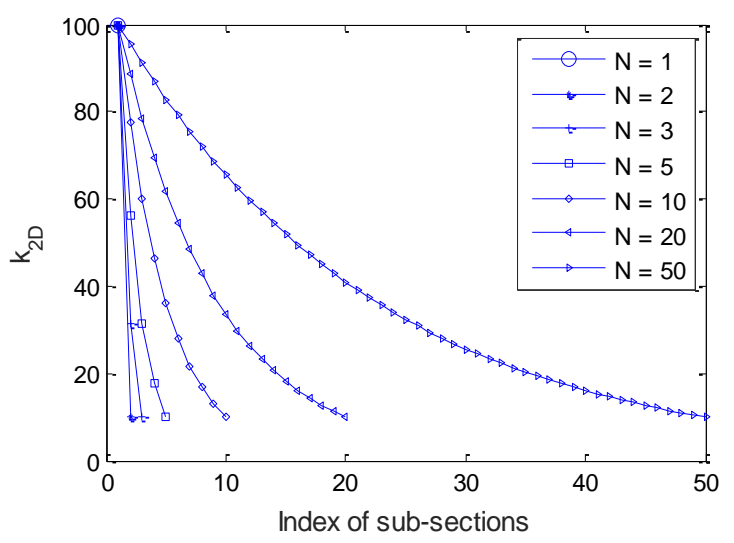

(b) logarithmic functions

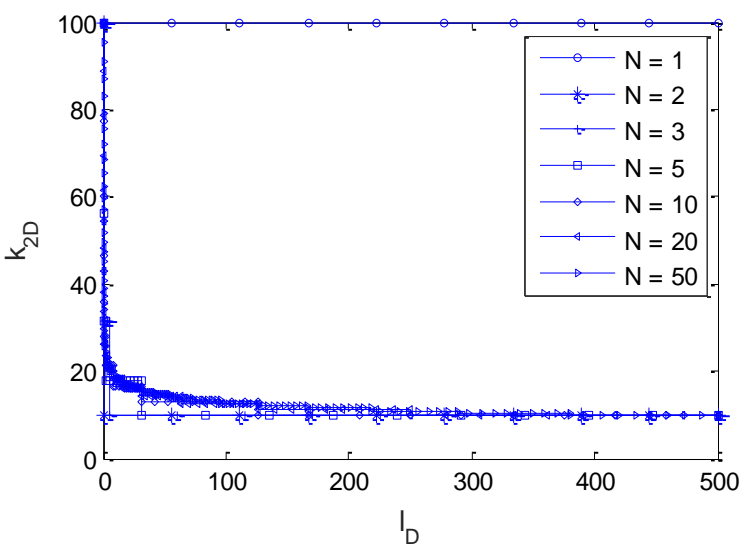

(d) logarithmic functions 


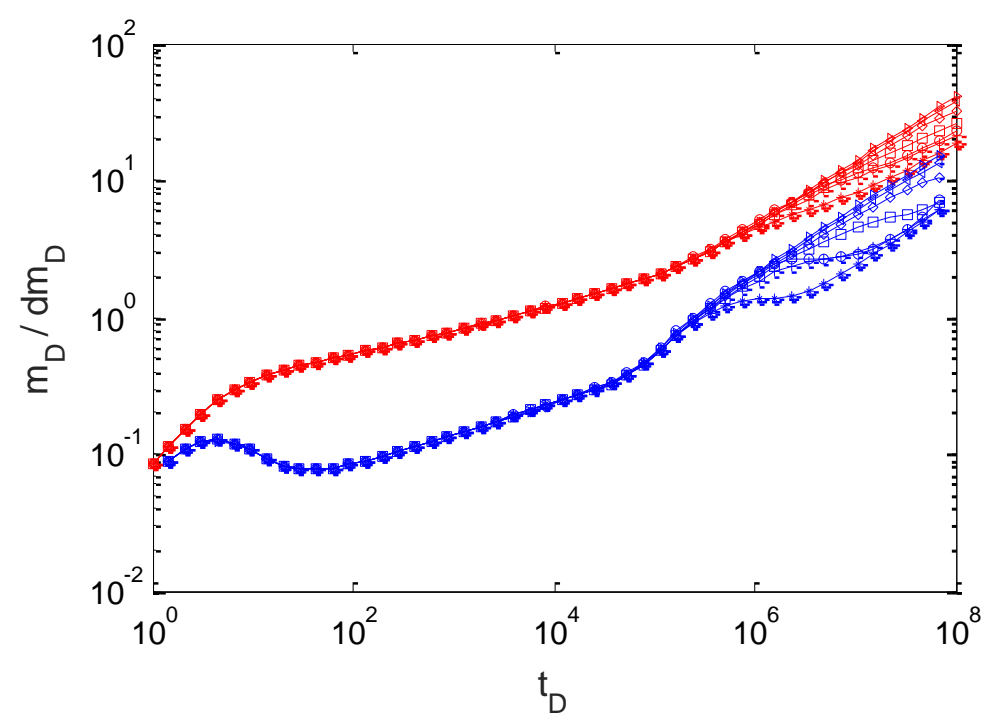

(e) linear function

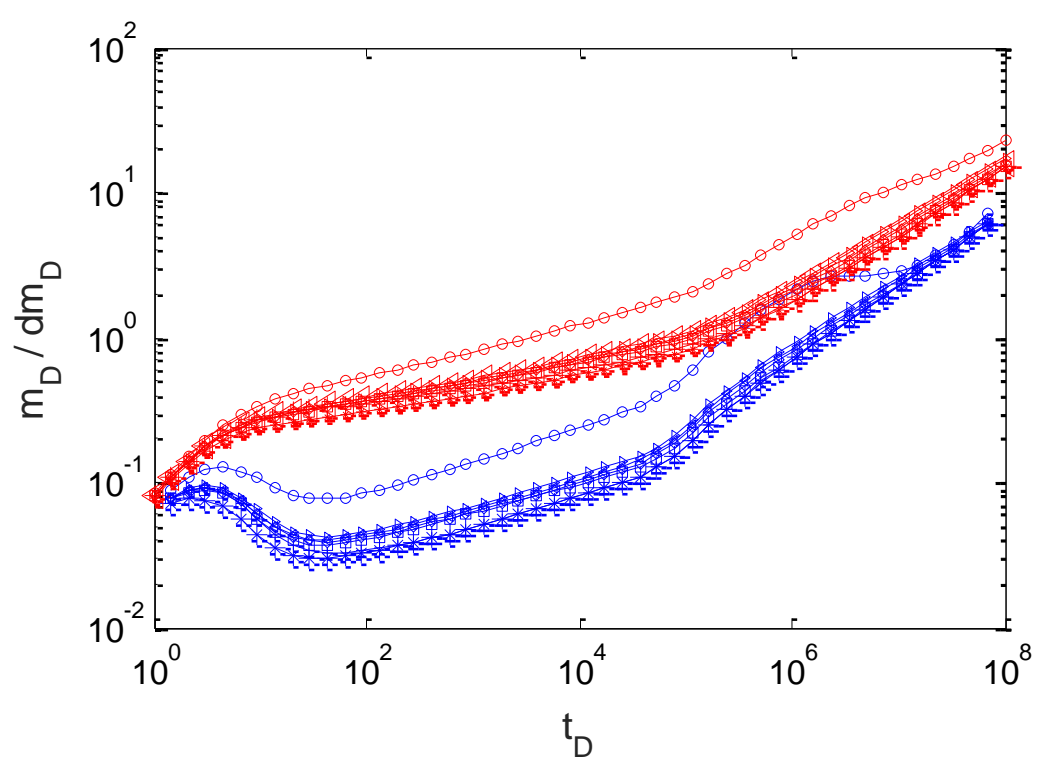

(f) logarithmic functions

Figure 10: The effect of coefficient of permeability variation on pressure and derivative curves

\section{Conclusion}

An analytical multi-linear model is proposed for multi-fractured horizontal wells with SRV in tight gas reservoirs. The transient pressure and pressure derivative curves are also established. The principal contributions in this work are summarized as follows:

(1) By using the Perturbation technique and Laplace transformation,with consideration the stress sensitivity and SRV is obtained. The solution of transient pressure behaviors for multi-fractured horizontal well with SRV in stress sensitive shale gas reservoirs is finally obtained by Stefest numerical inversion algorithm.

(2) This newly proposed mulit-linear flow model (MLFM) is validated by comparing with an existing model and a perfect agreement has been obtained.

(3) Approximately five flow stages can be identified: Wellbore storage and skin effect flow; The formation bin-linear flow in the inner SRV; The formation linear flow in the SRV 
zone and the transition flow between SRV zone II and zone IV; the transition flow between SRV zone II and zone III; The formation linear flow in zone III.

(4) SRV zone II provides the main flow path to supply gas from shale gas reservoir to wellbore. Increasing the size of outer-region could delay the negative influences of boundary conditions. The permeability of SRV zone III mainly impacts the formation linear flow regimes, while the permeability of zone IV has significant influences on the formation linear flow regime in SRV zone II.

(5) To systematically represent the spatial variation of permeability in Zone III, both linear and logarithmic functions are used to describe the reduction of permeability. In contrast to the linear function, logarithmic function yields a more rapid reduction of dimensionless permeability. As the number of subsections increases, the reduction rate of permeability will be decreases, as a result, the negative of boundary condition will be delayed. The logarithmic function easily leads to a rapid reduction of permeability and hence almost the entire flow regimes are severely influenced.

\section{Conflicts of Interest}

The author(s) declared no potential conflicts of interest with respect to the research, authorship, and/or publication of this article.

\section{Funding Statement}

The authors acknowledge the financial support provided by the National Natural Science Foundation of China(No. U1562102).

\section{References}

Clarkson, C.R, 2013. Production data analysis of unconventional gas wells: Review of the theory and best practices. Int. J,Coal Geoal.109-110,101-149.

Ozkan, E. Brown, M. and Raghavan, R, et al. Comparison of fractured horizontal well performance in tight sand and shale gas reservoirs.SPE Reservoir Evaluation \& Engineering,2011,14(2):248-259.

Ekaterina Stalgorova and Louis Mattar. Analytical Model for Unconventional Multifractured Composite Systems.August 2013 SPE Reservoir and Evaluation \& Engineering.SPE 162516.Presented at the SPE Canadian Unconventional Resource Conference,Calgary,30 October-1 November 2012.

Zhao, Y. L., Zhang, L. H., Luo, J. X, et al (2014). "Performance of fractured horizontal well with stimulated reservoir volume in unconventional gas reservoir". Journal of Hydrology, $512,447-456$.

Fatt, I., and Davis, D.H.. (1952). "Reduction in permeability with overburden pressure". Journal of Petroleum Technology, 4(12), 16.

Fatt, I. (1953). "The effect of overburden pressure on relative permeability". Journal of Petroleum Technology, 5(10), 15-16.

Gray, D. H. , and Fatt, I.. (1963). "The effect of stress on permeability of sandstone cores". Society of Petroleum Engineers Journal, 3(02), 95-100.

Vairogs J and Rhoades V W. (1973). "Pressure transient tests in formations having stresssensitive permeability". Journal of Petroleum Technology, 25(8):965-970.

Pedrosa, O. A. J. (1986). "Pressure transient response in stress-sensitive formations". Spe California Regional Meeting. pp:203-210.

Kilmer N H , Morrow N R , Pitman J K . (1987), "Pressure sensitivity of low permeability sandstones". Journal of Petroleum Science and Engineering, 1(1):65-81.

Gang Han and Marte Gutierrez and Peter Schmitz. (2013). "Stress-dependent Flow, Mechanical, and Acoustic Properties for an Unconventional Oil Reservoir Rock". 
Unconventional Resources Technology Conference, Denver, Colorado, pp. 12-14. August.

Ozkan, E., Brown, M. L. , Raghavan, R, et al. (2011). " Comparison of fractured-horizontalwell performance in tight sand and shale reservoirs". SPE Reservoir Evaluation \& Engineering, 14(02), 248-259.

Stalgorova, K. and Mattar, L, (2013). "Analytical model for unconventional multi-fractured composite systems". SPE Reservoir Eval. Eng. 16 (03),246-256.

Ji, J., Yao, Y., Huang, S., Ma, X., et al. (2017). "Analytical model for production performance analysis of multi-fractured horizontal well in tight oil reservoirs". Journal of Petroleum Science and Engineering, 158, 380-397.

Wu, ZW, Cui C, Lv G, et al (2019). "A multi-linear transient pressure model for multistage fractured horizontal well in tight oil reservoirs with considering threshold pressure gradient and stress sensitivity". Journal of Petroleum Science and Engineering, 172, 839-854.

Jiang, R., Xu, J., Sun, Z., et al. (2014). "Rate transient analysis for multistage fractured horizontal well in tight oil reservoirs considering stimulated reservoir volume". Mathematical Problems in Engineering, 2014.

Zhao, Y. L., Zhang, L. H., Xu, B. Q, et al. (2016). "Analytical solution and flow behaviors of horizontal well in stress-sensitive naturally fractured reservoirs". International Journal of Oil, Gas and Coal Technology, 11(4), 350-369.

Jia, P. , Cheng, L. , Huang, S. , et al. (2017). “A comprehensive model combining Laplacetransform finite difference and boundary-element method for the flow behavior of a twozone system with discrete fracture network". Journal of Hydrology, 551, 453-469.

Wang, J, Jia, A, Wei, Y, et al. (2018). "Semi-analytical simulation of transient flow behavior for complex fracture network with stress-sensitive conductivity". Journal of Petroleum Science and Engineering, 171, 1191-1210.

$\mathrm{Xu}$, J., Guo, C., Wei, M.et al (2015). "Production performance analysis for composite shale gas reservoir considering multiple transport mechanisms". Journal of Natural Gas Science and Engineering, 26, 382-395.

Ren Z.X, Xiaodong, W, Dandan, L., Rui, R., Wei, G., Zhiming, C. \& Zhaoguang, T. (2016). "Semi-analytical model of the transient pressure behavior of complex fracture networks in tight oil reservoirs". Journal of Natural Gas Science and Engineering, 35, 497-508.

Warren, J. E and Root, P. J. (1963). "The behavior of naturally fractured reservoirs". Society of Petroleum Engineers Journal, 3(03), 245-255.

Liu, M., Xiao, C., Wang, Y., et al. (2015). "Sensitivity analysis of geometry for multi-stage fractured horizontal wells with consideration of finite-conductivity fractures in shale gas reservoirs". Journal of Natural Gas Science and Engineering, 22, 182-195.

Chen, Z., Liao, X., Yu, W.et al. (2018). "Pressure-Transient Behaviors of Wells in Fractured Reservoirs With Natural-and Hydraulic-Fracture Networks". SPE Journal.

Stehfest, H., 1970. "Numerical inversion of Laplace transforms". Commun. ACM 13 (1), 4749.

Ren, Z.X., Wu, X.D., Han, G.Q., et al. (2017). “ Transient pressure behavior of multi-stage fractured horizontal wells in stress-sensitive tight oil reservoirs". Journal of Petroleum Science and Engineering, 157, 1197-1208.

Agarwal, R.G. and Al-Hussainy and R., Ramey Jr, H. J. (1970) An investigation of wellbore storage and skin effect in unsteady liquid flow: I. Analytical treatment. Society of Petroleum Engineers Journal, 10(03), 279-290

Kuchuk, F. J., Onur, M., Hollaender, F. (2010). Pressure transient formation and well testing: convolution, deconvolution and nonlinear estimation (Vol. 57). Elsevier.

Song, B. and Economides, M. J. and Ehlig-Economides, C. A. (2011, January). Design of 
multiple transverse fracture horizontal wells in shale gas reservoirs. In SPE Hydraulic Fracturing Technology Conference. Society of Petroleum Engineers.

Jiahang, Wang, W. Xiaodong, and D. Wenxiu . "A Semianalytical Model for MultipleFractured Horizontal Wells with SRV in Tight Oil Reservoirs." Geofluids 2017(1):1-15. Hai-Tao Wang.Performance of multiple fractured horizontal wells in shale gas reservoirs with consideration of multiple mechanisms. Journal of Hydrology,2013.

El- Banbi, AH. 1998. Analysis of Tight Gas Wells. PhD. Dissertation, Texas.

Hasan A. Al-Ahmadi, 2011. SPE Triple porosity Models: One Future Step Towards Capturing Fractured Reservoirs Heterogeneity.

Bingxiang Xu, Manouchehr Haghighi, Xiangfang Li, Dennis Cooke. Development of new type curves for production analysis in naturally fractured shale gas/tight gas reservoirs. Journal of Petroleum Science and Engineering 105(2013):107-115. 